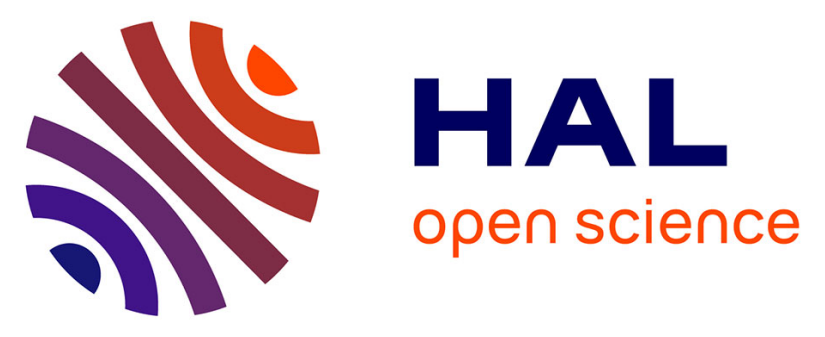

\title{
Control of unstable delayed recycling systems
} Rocio Jasmin Vazquez-Guerra, Juan Francisco Marquez-Rubio, David Fernando Novella-Rodríguez, Basilio del Muro-Cuellar, Domingo Cortes-Rodríguez

\section{- To cite this version:}

Rocio Jasmin Vazquez-Guerra, Juan Francisco Marquez-Rubio, David Fernando Novella-Rodríguez, Basilio del Muro-Cuellar, Domingo Cortes-Rodríguez. Control of unstable delayed recycling systems. Asia-Pacific Journal of Chemical Engineering, 2019, pp.e2290. 10.1002/apj.2290 . hal-02025669

\section{HAL Id: hal-02025669 https://hal.science/hal-02025669}

Submitted on 19 Feb 2019

HAL is a multi-disciplinary open access archive for the deposit and dissemination of scientific research documents, whether they are published or not. The documents may come from teaching and research institutions in France or abroad, or from public or private research centers.
L'archive ouverte pluridisciplinaire HAL, est destinée au dépôt et à la diffusion de documents scientifiques de niveau recherche, publiés ou non, émanant des établissements d'enseignement et de recherche français ou étrangers, des laboratoires publics ou privés. 


\title{
Control of unstable delayed recycling systems
}

\author{
R.J. Vazquez Guerra*, J.F. Marquez Rubio*, \\ D.F. Novella Rodríguez**, B. Del Muro Cuéllar*, \\ D. Cortés Rodríguez \\ * Instituto Politécnico Nacional, \\ Escuela Superior de Ingeniería Mecánica y Eléctrica, \\ Unidad Culhuacan, México D.F., 04430, \\ México. \\ E-mail: rjjvg@yahoo.com.mx, jfcomr23@yahoo.com.mx, \\ bdelmuro@yahoo.com,domingo.cortes@gmail.com \\ ** Univ. Grenoble Alpes, GIPSA-lab, Grenoble, France. \\ E-mail: dnovellar@gmail.com
}

\begin{abstract}
This work deals with the stabilization of recycling systems with unstable dynamics at both, the direct path and the recycling paths as well as different timedelays for each path. The main contribution of this work is to provide a stabilizing strategy to the mentioned class of systems using an observer based control approach. Stability results concerning stable recycling systems or partially unstable recycling system can be found in literature. However, to the best of our knowledge, there is not stability results when unstable dynamics are considered in both paths of the recycling process. The provided necessary and sufficient stability conditions of the proposed stabilizing controllers are obtained from a frequency domain analysis.
\end{abstract}

Key Words: Linear Systems, time-delay, unstable, recycling.

\section{Introduction}

Recycling systems are commonly found in chemical industry, for instance, in a typical plant formed by reactor/separator process, where reactants are recycled back to the reactor [1]. This kind of systems reuses the energy and the partially processed matter increasing the efficiency of the overall process. In recycling systems, a partial feedback of the process output to the input yields a model with positive feedback, which can give rise to some undesirable effects. In [2] the effects of recycle path on dynamics process and their implications to plant-wide control were studied. In [3], robust control for recycling plants was discussed and a scheme of recycle compensation to eliminate the effects of the recycle was proposed. Similar approaches were employed in [4-6].

When in addition to the recycle path a time-delay is present, exponential terms appear in the direct and the recycling paths. In a state space representation, recycling systems with time-delay can 
be related to systems with delays in the input and the state variables. In [7], an approximated model to represent recycling systems by using discrete-time approach was proposed. Such approximated models can be used for stability analysis or control design [4, 8-10].

In general, unstable delayed systems are more difficult to control than stable delayed systems. For instance, the original Smith Predictor cannot be used in the case of delayed unstable processes. However, many works have proposed modifications to the original Smith Predictor in order to deal with unstable systems, see for instance [11]. If a recycle is added in such unstable delayed system would lead to a more difficult (although interesting) problem. Existing Smith Predictor based schemes cannot directly be applied to this kind of systems. This is, because the open-loop recycling system is not just a system with one time-delay in the direct path but it is a system with an internal delay term, i.e., an open-loop infinite dimensional system. To tackle this problem, we consider here recycling systems composed of a plant with one unstable pole, several stable poles and a delay term in the direct path and the same type of delayed unstable subsystem (with possibly different delay magnitude) in the recycling path.

The present work extends the results presented in $[12,13]$ where recycling systems are restricted to be unstable first order plants in the direct path. A first attempt to extend these results was presented in [14], dealing with a high order plant in the direct path with one unstable pole and a stable recycling subsystem. Results developed in [14] do not achieve step tracking or step disturbance rejection.

There are stability results to stable recycling systems or partially unstable recycling system. However, to the best of our knowledge there is not stability results when unstable dynamics are considered in both paths of the recycling process. In this work, a control scheme is proposed in order to stabilize and control the delayed recycling processes previously depicted. The proposed controller allows to achieve the closed-loop stability. Then, necessary and sufficient conditions are given for the existence of the proposed stabilizing scheme, which are obtained from a frequency domain approach. It is important to note that, the problem of the stabilization and control of delayed unstable plants even without recycle path is not completely solved, at least from the classical controllers P, PI, PD and PID viewpoint. For instance, recent works deal with the stabilization and control of delayed systems with one [15-17] and two [18] unstable pole(s).

The main contribution of the work is that the stability step into the proposed observer based scheme considers the existence of the recycling path, this feature is not considered in some works, see for instance [2]-[6],[12]-[15],[19]. These mentioned works intend to eliminate the recycling dynamic in order to design a controller considering only to the direct path. Thus, when modelling uncertainties appears into the recycling path, the removal stage preserves the characteristic of the nominal system and some troubles may arises with respect to closed-loop stability/robustness. The proposed methodology in this manuscript does not intend to cancel recycling dynamics. Instead, under the knowledge of a special internal variable system, a control strategy is designed taking into account the whole system consisting in direct and recycling paths. This property allows to improve robustness issues considerably and this feature is precisely the key point to consider unstable dynamics at recycling path. However, the use of the proposed control scheme is not limited to unstable dynamics at recycling, i.e., the proposed result can also be used in stable recycling systems.

The outline of the paper is as follows. In Section 2 the problem is formulated and the class of systems considered is stated. The general idea of the solution is also outlined in this section, namely the need of an observer strategy. In Section 3 some preliminary results are presented. The Section 4 
presents the main results. Some numerical examples are described in Section 5. Finally Section 6 presents some conclusions.

\section{Problem formulation}

Consider the class of recycling system (see Figure 1) described

$$
Y(s)=\left[\begin{array}{ll}
G_{d}(s) & G_{d}(s) G_{r}(s)
\end{array}\right]\left[\begin{array}{c}
U(s) \\
Y(s)
\end{array}\right],
$$

with,

$$
\begin{aligned}
& G_{d}(s)=G_{1}(s) e^{-\tau_{1} s}=\frac{\alpha}{D_{1}(s)} e^{-\tau_{1} s} \\
& G_{r}(s)=G_{2}(s) e^{-\tau_{2} s}=\frac{\beta}{D_{2}(s)} e^{-\tau_{2} s}
\end{aligned}
$$

where $D_{1}(s)=(s-a)\left(s+b_{1}\right) \ldots\left(s+b_{m}\right)$ and $D_{2}(s)=(s-c)\left(s+d_{1}\right) \ldots\left(s+d_{n}\right) . G_{d}(s)$ and $G_{r}(s)$ are transfer functions of the direct and the recycling paths, respectively; $\tau_{1}, \tau_{2} \geq 0$ are the timedelays associated to $G_{d}(s)$ and $G_{r}(s) . a, b_{i}, c, d_{j} \in \mathbb{R}^{+}$with $i=1,2, \ldots, m$ and $j=1,2, \ldots, n$, i.e., $G_{d}(s)$ and $G_{r}(s)$ are unstable. $U(s)$ and $Y(s)$ are the input and the output signals, respectively.

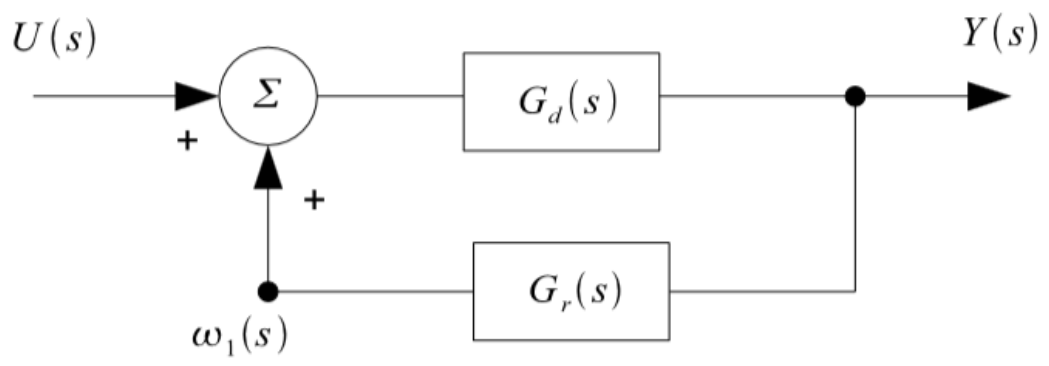

Figure 1: A process with recycle

The transfer function of the recycling system (1) is given by,

$$
\frac{Y(s)}{U(s)}=\frac{\alpha D_{2}(s) e^{-\tau_{1} s}}{D_{1}(s) D_{2}(s)-\alpha \beta e^{-\left(\tau_{1}+\tau_{2}\right) s}} .
$$

Note that exponential terms appear explicitly in the numerator and the denominator of (3). Stability of (3) is determined by the roots of its characteristic quasi-polynomial,

$$
Q(s)=D_{1}(s) D_{2}(s)-\alpha \beta e^{-\left(\tau_{1}+\tau_{2}\right) s} .
$$

The transcendental term in $Q(s)$ causes an infinite number of roots. For this kind of plants it is not an easy task to conclude something about the dynamical behaviour (stability for instance) even in the case of uncontrolled plant. Obviously, the related transfer function when the system is controlled with an output feedback becomes more complicated involving more than one transcendental term.

Let us to describe some initial ideas. With reference to Figure 1, if the signal $\omega_{1}(s)$ were measured, then we could set, 


$$
U(s)=\left(R(s)-\omega_{1}(s)\right) C(s),
$$

obtaining the system shown in Figure 2, where $R(s)$ is the input reference and $C(s)$ is the controller. Then, it would be possible to design the controller, $C(s)$, such that the closed-loop system is stable. In fact, the main aims of this work can be summarized as follows:

i) Finding the stability conditions of the proposed feedback shown in Figure 2.

ii) Since $\omega_{1}(s)$ is assumed as an unmeasured internal system signal, an observer scheme to estimate this variable is developed in the Section 4.

In the next section some preliminary stability results are presented.

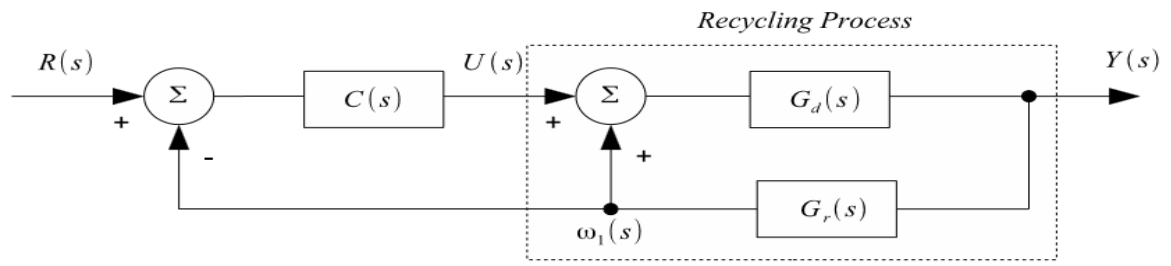

Figure 2: Recycling delayed process with the proposed control law (5)

\section{Preliminary stability results}

Consider the system,

$$
\frac{Y(s)}{U(s)}=\frac{\gamma}{(s-a)\left(a+b_{1}\right)\left(a+b_{2}\right) \ldots\left(a+b_{p}\right)} e^{-\tau s},
$$

$a, b_{i} \in \mathbb{R}$, with $a, b_{i}>0 \forall i=1,2, \ldots, p ; \frac{1}{a}-\sum_{i=1}^{m} \frac{1}{b_{i}}>0$ and the static control law,

$$
U(s)=(R(s)-Y(s)) k \text {, }
$$

with $R(s)$ as the input reference. The following result provides a stability condition when a proportional controller is used for unstable delayed systems including one unstable pole, $p$ stable poles and a time-delay.

Lemma 1 [16,20] There exists a gain $k$ such that the closed-loop system,

$$
\frac{Y(s)}{R(s)}=\frac{k \gamma e^{-\tau s}}{(s-a)\left(s+b_{1}\right)\left(s+b_{2}\right) \ldots\left(s+b_{p}\right)+k \gamma e^{-\tau s}},
$$

is stable if and only if

$$
\tau<\frac{1}{a}-\sum_{i=1}^{p} \frac{1}{b_{i}}
$$

From condition (9), the size of the delay to achieve the closed-loop stability is restricted by the position of the open-loop unstable pole and the stable poles in the real axis of the plane $s$.

Let us now find the set of stabilizing gains $k$ related to Lemma 1 is obtained. The phase crossover frequency $\omega_{c}$ is given by,

$$
\begin{gathered}
-\tau \omega_{c}+\arctan \left(\frac{\omega_{c}}{a}\right)-\sum_{i=1}^{m} \arctan \left(\frac{\omega_{c}}{b_{i}}\right)=0, \\
4
\end{gathered}
$$


where $\omega_{c}>0$. Then, the stabilizing gain $k$ is within the interval of,

$$
\frac{a}{\gamma} \prod_{i=1}^{m} b_{i}<k<\frac{a}{\gamma} \prod_{i=1}^{m} b_{i} M
$$

with

$$
M=\sqrt{\left(1+\left(\frac{\omega_{c}}{a}\right)^{2}\right) \prod_{i=1}^{m}\left(1+\left(\frac{\omega_{c}}{b_{i}}\right)^{2}\right)}
$$

In what follows the stability condition of a particular output injection schema is provided. This proposed schema will be used later to design the proposed observer strategy.

Consider the recycling system given by (1)-(2). A state space representation of $G_{1}(s)$ as $\left(A_{d}\right.$, $\left.B_{d}, C_{d}\right)$ is given by,

$$
A_{d}=\left[\begin{array}{ccccc}
a & 0 & 0 & \cdots & 0 \\
1 & -b_{1} & 0 & \cdots & 0 \\
0 & 1 & -b_{2} & \cdots & 0 \\
\vdots & \vdots & \vdots & \ddots & \vdots \\
0 & 0 & 0 & \cdots & -b_{m}
\end{array}\right], B_{d}=\left[\begin{array}{c}
\alpha \\
0 \\
0 \\
0 \\
0
\end{array}\right], C_{d}=\left[\begin{array}{lllll}
0 & 0 & 0 & \cdots & 1
\end{array}\right]
$$

A state space representation of $G_{2}(s)$ as $\left(A_{r}, B_{r}, C_{r}\right)$ with,

$$
A_{r}=\left[\begin{array}{ccccc}
c & 0 & 0 & \cdots & 0 \\
1 & -d_{1} & 0 & \cdots & 0 \\
0 & 1 & -d_{2} & \cdots & 0 \\
\vdots & \vdots & \vdots & \ddots & \vdots \\
0 & 0 & 0 & \cdots & -d_{n}
\end{array}\right], B_{r}=\left[\begin{array}{c}
1 \\
0 \\
0 \\
0 \\
0
\end{array}\right], C_{r}=\left[\begin{array}{lllll}
0 & 0 & 0 & \cdots & \beta
\end{array}\right],
$$

and the injection vectors defined by $L_{1}=\left[\begin{array}{llll}l_{1-(1)} & l_{1-(2)} & \cdots & l_{1-(m+1)}\end{array}\right], L_{2}=\left[\begin{array}{llll}\bar{l}_{2-(1)} & 0 & \cdots & 0\end{array}\right]^{T}$. Therefore, a state space representation of the closed-loop system $\frac{\bar{Y}(s)}{\bar{R}(s)}$ related to the control scheme presented in Figure 3 can be written as,

$$
\begin{gathered}
\dot{x}(t)=\bar{A} x(t)+\bar{A}_{1} x(t-\theta)+\bar{B} \bar{r}\left(t-\tau_{1}\right) \\
y(t)=\bar{C} x(t)
\end{gathered}
$$

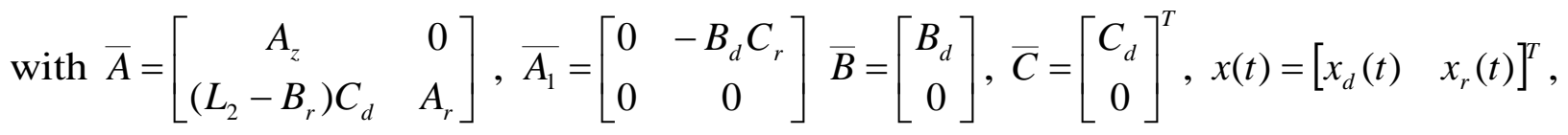
$A_{z}=A_{d}-L_{1} C_{d}$, and $\theta=\tau_{1}+\tau_{2}$

Lemma 2. Consider the recycling system given by (1)-(2), and the output injection strategy shown in Figure 3. There exists a vector $L=\left[\begin{array}{ll}L_{1} & L_{2}\end{array}\right]^{T}$ such that the closed-loop transferfunction, $\frac{\bar{Y}(s)}{\bar{R}(s)}$ or equivalently (14)-(15) is stable if and only if, 


$$
\theta<\frac{1}{c}-\sum_{i=1}^{n} \frac{1}{d_{i}}
$$

with $\theta=\tau_{1}+\tau_{2}$.

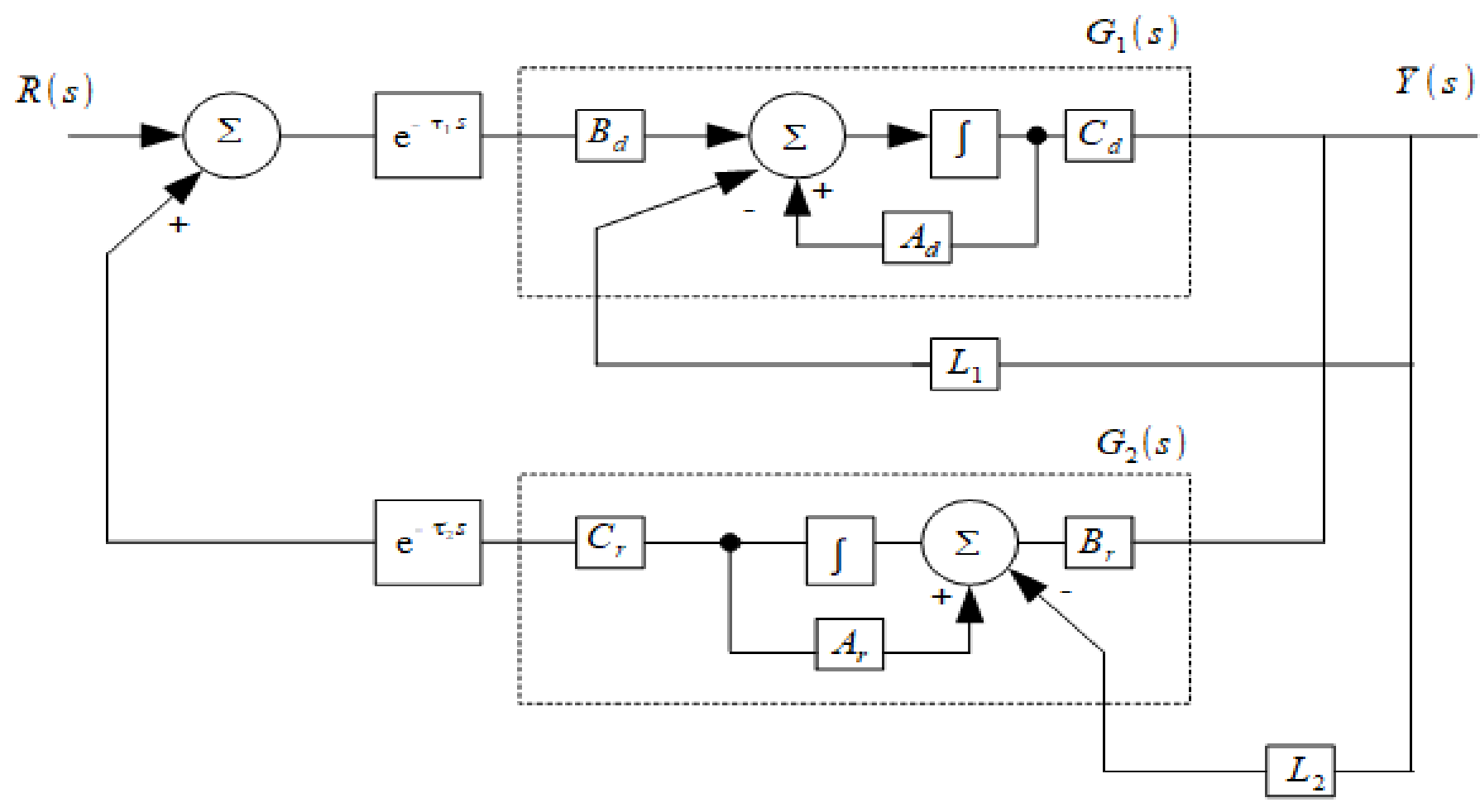

Figure 3: Output injection schema.

Proof. Consider the state space representation of $G_{1}(s)$ given by (12) and the partial output injection through $L_{1}$. Then, $L_{1}$ should be computed such that,

$$
\sigma\left(s I-A_{d}+L_{1} C_{d}\right)=\left\{-Z_{1},-Z_{2}, \ldots,-Z_{m+1}\right\}
$$

where $\sigma(*)$ represents the eigenvalues of $*$, and $-z_{1},-z_{2}, \ldots,-z_{m+1}$ are the relocated stable poles of $G_{1}(s)$. i.e., $A_{z}=A_{d}-L_{1} C_{d}$ and it can be written as,

$$
A_{z}=\left[\begin{array}{ccccc}
-z_{1} & 0 & 0 & \cdots & 0 \\
1 & -z_{2} & 0 & \cdots & 0 \\
0 & 1 & -z_{3} & \cdots & 0 \\
\vdots & \vdots & \vdots & \ddots & \vdots \\
0 & 0 & 0 & & -z_{m+1}
\end{array}\right]
$$

Consider also that the state space representation of $G_{2}(s)$ given by (13) yielding to the closedloop representation given by (14)-(15).

Notice that the term $\left(L_{2}-B_{r}\right) C_{d}$ in equation (14) is a free term due to the gain $L_{2}$ is the control parameter. From (14)-(15), it is possible to obtain the closed-loop transfer function $\frac{\bar{Y}(s)}{\bar{R}(s)}$ which results, 


$$
\frac{\bar{Y}(s)}{\bar{R}(s)}=\bar{C}\left(s I-\bar{A}-\bar{A}_{1} e^{-\theta s}\right)^{-1} \bar{B} e^{-\tau_{1} s},
$$

where $I$ is the identity matrix. The closed-loop stability depends on the characteristic equation associated to the the transfer function (18), which is given by,

$$
\operatorname{det}\left(s I-\bar{A}-\bar{A}_{1} e^{-\theta s}\right)=0
$$

or equivalently,

$$
(s-c)\left(s+d_{1}\right)\left(s+d_{2}\right) \ldots\left(s+d_{n}\right)\left(s+z_{1}\right)\left(s+z_{2}\right) \ldots\left(s+z_{m+1}\right)+K \alpha \beta e^{-\theta s}=0
$$

where $K=1-\bar{l}_{2-(1)}$, and $\bar{l}_{2-(1)}$ is a free static gain. From (19), the closed-loop stability of the proposed output injection is reduced to a system of the form (8), where its stability properties are analyzed in Lemma 1. In this way the associated stability condition is obtained as,

$$
\theta<\frac{1}{c}-\sum_{i=1}^{n} \frac{1}{d_{i}}-\sum_{i=1}^{m+1} \frac{1}{z_{i}}
$$

However, the stability condition (20) can be improved by choosing $L_{1}$ such that the relocated poles of $G_{1}(s),\left\{-z_{1},-z_{2}, \ldots,-z_{p}\right\}$ are sufficiently far from the origin i.e., $-z_{1},-z_{2}, \ldots,-z_{p} \rightarrow \infty$. Giving as result that condition (20) becomes $\theta<\frac{1}{c}-\sum_{i=1}^{n} \frac{1}{d_{i}}-$ Hence the result follows.

Lemma 3. [21, 22] Given the open-loop transfer function $G(s)$ defined by:

$$
G(s)=\frac{N(s)}{D(s)} e^{-\tau s},
$$

a necessary condition for the closed-loop stability (when a unitary feedback is considered) is that the polynomial H(s):

$$
H(s)=\frac{\frac{d^{m+1}}{d s^{m+1}}\left(s^{v} D(s) e^{\tau s}\right)}{e^{\tau s}},
$$

has all its zeros in the open left half plane, where $m$ is the degree of $N(s)$ and $v$ is the systemtype, defined by the order of the integer in the open-loop transfer function.

\section{Main Results}

\subsection{Observer strategy}

In order to estimate the signal $\omega_{1}(s)$, in Figure 1, we propose the observer-predictor depicted in Figure 4. The convergence properties are established in the following result.

Theorem 1 Consider the observer scheme shown in Figure 4, assuming $G_{r}(s)$ and $G_{d}(s)$ defined by (2).

There exists a vector $L=\left[\begin{array}{ll}L_{1} & L_{2}\end{array}\right]^{T}$ such that

$$
\lim _{t \rightarrow \infty}\left[\omega_{1}(t)-\hat{\omega}_{1}(t)\right]=0
$$

if and only if 


$$
\theta<\frac{1}{c}-\sum_{i=1}^{n} \frac{1}{d_{i}}
$$

for $\theta=\tau_{1}+\tau_{2}$.

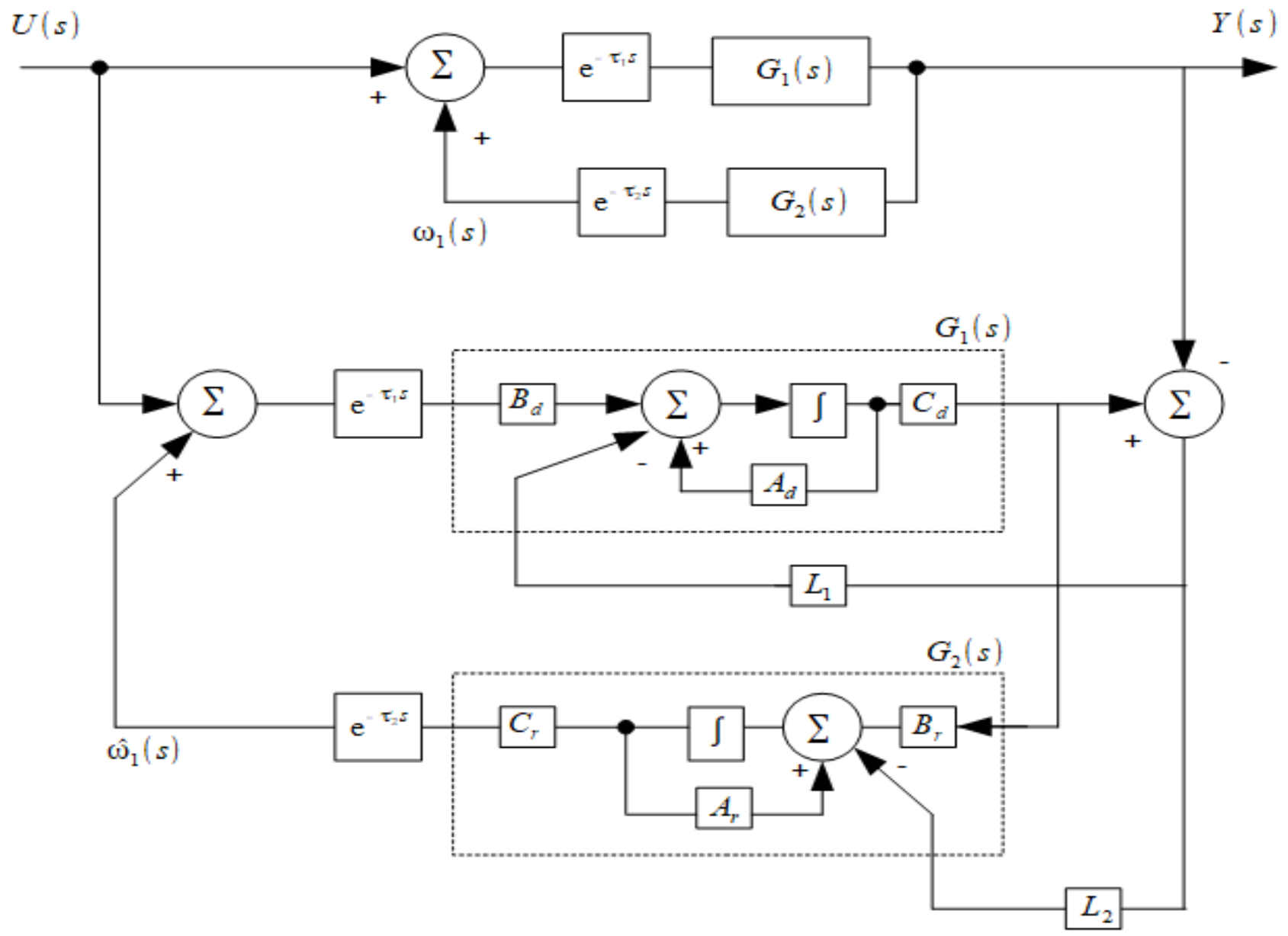

Figure 4: Proposed observer-predictor schema.

Proof. A state space representation of the observer-predictor scheme shown in Figure 4 is,

$$
\begin{gathered}
\dot{x}(t)=A x(t)+A_{1} x(t-\theta)+B u\left(t-\tau_{1}\right) \\
y(t)=C x(t)
\end{gathered}
$$

with, $\theta=\tau_{1}+\tau_{2}, x(t)=\left[\begin{array}{llll}x_{d}(t) & x_{r}(t) & \hat{x}_{d}(t) & \hat{x}_{r}(t)\end{array}\right]^{T}, y(t)=\left[\begin{array}{lll}y(t) & \hat{y}(t)\end{array}\right]^{T}, B=\left[\begin{array}{llll}B_{d} & 0 & B_{d} & 0\end{array}\right]^{T}$,

$$
A=\left[\begin{array}{cccc}
A_{d} & 0 & 0 & 0 \\
B_{r} C_{d} & A_{r} & 0 & 0 \\
L_{1} C_{d} & 0 & A_{d}-L_{1} C_{d} & 0 \\
L_{2} C_{d} & B_{r} C_{d}-L_{2} C_{d} & -L_{2} C_{d} & A_{r}
\end{array}\right], A_{1}=\left[\begin{array}{cccc}
0 & B_{d} C_{r} & 0 & 0 \\
0 & 0 & 0 & 0 \\
0 & 0 & 0 & B_{d} C_{r} \\
0 & 0 & 0 & 0
\end{array}\right], C=\left[\begin{array}{cccc}
C_{d} & 0 & 0 & 0 \\
0 & 0 & C_{d} & 0
\end{array}\right],
$$

where $x(t) \in R^{m+n+2}$ is the state vector, $u(t) \in R$ is the input, $y(t) \in R^{2}$ is the output, $\tau_{1} \geq 0$ and 
$\tau_{2} \geq 0$ are the constant time-delays in the system. $A_{d} \in R^{(m+1) x(m+1)}, B_{d} \in R^{(m+1) x 1}$, and $C_{d} \in R^{1 x(m+1)}$ are matrices and vectors parameters that correspond to the direct path in the process, $G_{1}(s)$ is represented by,

$$
A_{d}=\left[\begin{array}{ccccc}
a & 0 & 0 & \cdots & 0 \\
1 & -b_{1} & 0 & \cdots & 0 \\
0 & 1 & -b_{2} & \cdots & 0 \\
\vdots & \vdots & \vdots & \ddots & \vdots \\
0 & 0 & 0 & \cdots & -b_{m}
\end{array}\right], B_{d}=\left[\begin{array}{c}
\alpha \\
0 \\
0 \\
0 \\
0
\end{array}\right], C_{d}=\left[\begin{array}{lllll}
0 & 0 & 0 & \cdots & 1
\end{array}\right],
$$

and $A_{r} \in R^{(n+1) x(n+1)}, B_{r} \in R^{(n+1) x 1}$, and $C_{r} \in R^{1 x(n+1)}$ are matrices and vectors parameters that corresponds to the recycling path in the process, $G_{2}(s)$ is represented by,

$$
A_{r}=\left[\begin{array}{ccccc}
c & 0 & 0 & \cdots & 0 \\
1 & -d_{1} & 0 & \cdots & 0 \\
0 & 1 & -d_{2} & \cdots & 0 \\
\vdots & \vdots & \vdots & \ddots & \vdots \\
0 & 0 & 0 & \cdots & -d_{n}
\end{array}\right], B_{r}=\left[\begin{array}{c}
1 \\
0 \\
0 \\
0 \\
0
\end{array}\right], C_{r}=\left[\begin{array}{lllll}
0 & 0 & 0 & \cdots & \beta
\end{array}\right],
$$

$\hat{x}(t)$ is the estimation of $x(t)$.

From Figure 4, we are strictly interested in the convergence of $e_{\omega_{1}}(t)=\hat{\omega}_{1}(t)-\omega_{1}(t)$. However, as $\omega_{1}(t)$ is a static function of the state $x_{r}(t)$ and it also depends on $e_{x d}(t)$, then, we can equivalently analyze the convergence of $e_{x d}(t)$ and $e_{x r}(t)$ defined in what follows. Defining the stateprediction errors $e_{x d}(t)=\hat{x}_{d}(t)-x_{d}(t)$ and $e_{x r}(t)=\hat{x}_{r}(t)-x_{r}(t)$, it is possible to describe the behavior of the error signals as,

$$
e(t)=\left[\begin{array}{cc}
A_{d}-L_{1} C_{d} & 0 \\
\left(L_{2}-B_{r}\right) C_{d} & A_{r}
\end{array}\right] e(t)+\left[\begin{array}{cc}
0 & -B_{d} C_{r} \\
0 & 0
\end{array}\right] e(t-\theta)
$$

with, $e(t)=\left[e_{x d}(t) e_{x r}(t)\right]$. Notice that the term $\left(L_{2}-B_{r}\right) C_{d}$ in (28) is a free term due to the gain $L_{2}$ is the control parameter.

On the other hand, consider the closed-loop system analyzed in Lemma 2, given by (14)-(15). In this way, comparing (28) and (14) it is clear that Lemma 2 can be applied to system (28). Hence the result of the theorem follows.

To find the observer parameters, the following procedure is suggested.

\section{Procedure 1}

1. Verify the necessary and sufficient condition in Theorem 1 expressed in (24) is satisfied.

2. Tocompute the observer parameter $L_{1}$. Consider a traditional output injection to the transfer function $G_{1}(s)$ and propose the relocated poles of the $G_{1}(s)$ such that $z_{1}, z_{2}, \ldots, z_{m+1} \rightarrow \infty$. In this way, the following expression should be solved to $L_{1}$,

$$
\operatorname{det}\left(s I-A_{d}+L_{1} C_{d}\right)=\left(s+z_{1}\right)\left(s+z_{2}\right) \ldots\left(s+z_{m+1}\right)
$$

with $A_{d}, C_{d}$ as given in (27). Also the observer parameter $L_{1}$ can be obtained using the Ackerman formulation. 
3. By applying the results of Lemma 1 to the case of our interest, the parameter $\bar{l}_{2-(1)}$ can be obtained from

$$
1+\frac{c}{\alpha \beta} \prod_{i=1}^{n} d_{i} \prod_{i=1}^{m+1} z_{i}<\bar{l}_{2-(1)}<1+\frac{c}{\alpha \beta} M \prod_{i=1}^{n} d_{i} \prod_{i=1}^{m+1} z_{i}
$$

with

$$
M=\sqrt{\left(1+\frac{\omega_{c}{ }^{2}}{c^{2}}\right) \prod_{i=1}^{n}\left(1+\frac{\omega_{c}{ }^{2}}{d_{i}{ }^{2}}\right) \prod_{i=1}^{m+1}\left(1+\frac{\omega_{c}{ }^{2}}{z_{i}{ }^{2}}\right)} .
$$

and $\omega_{c}$ is obtained bysolving, where $\omega_{c}>0$.

$$
-\theta \omega_{c}+\arctan \left(\frac{\omega_{c}}{c}\right)-\sum_{i=1}^{n} \arctan \left(\frac{\omega_{c}}{d_{i}}\right)-\sum_{i=1}^{m+1} \arctan \left(\frac{\omega_{c}}{z_{i}}\right)=0,
$$

where $\omega_{c}>0$.

\subsection{Proposed Control Strategy}

In order to implement the ideas developed in Section 2 let us asume that an estimation of the signal $\omega_{1}(t)$ is taken from the observer designed in Subsection 4.1. In this way, in the following developments the stability properties of the closed-loop system shown in Figure 2 are analyzed. The following result presents the stability properties for the closed-loop system shown in Figure 2 when a Proportional-Derivative (PD) controller is considered. A frequency domain analysis is employed. Later, a Proportional-Integral-Derivative (PID) control is also considered to stabilize the closed-loop system.

Theorem 2 Consider the feedback control shown in Figure 2. Then, the delayed recycling system given by (1) can be stabilized with a PD controller given by,

$$
C(s)=K_{P}+K_{D} s,
$$

or

$$
C(s)=k_{p}\left(k_{d} s-1\right)+1,
$$

with $k_{p}, k_{d}>0$, if and only if,

$$
\theta<\frac{1}{a}+\frac{1}{c}-\sum_{i=1}^{m} \frac{1}{b_{i}}-\sum_{i=1}^{n} \frac{1}{d_{i}}-\sqrt{\frac{1}{a^{2}}+\frac{1}{c^{2}}+\sum_{i=1}^{m} \frac{1}{b_{i}^{2}}+\sum_{i=1}^{n} \frac{1}{d_{i}^{2}}}
$$

with $\theta=\tau_{1}+\tau_{2}$.

Proof. Sufficiency The closed-loop transfer function of the system shown in Figure 2 is given by,

$$
\frac{Y(s)}{R(s)}=\frac{C(s) G_{d}(s)}{1-(1-C(s)) G_{d}(s) G_{r}(s)} .
$$


and the associated closed-loop characteristic equation is,

$$
1+\bar{C}(s) G_{d}(s) G_{r}(s)=0
$$

with $\bar{C}(s)=C(s)-1$. The characteristic equation (36) can also be obtained from the auxiliary closed-loop system consisting in an open-loop transfer function $Q(s)=\bar{C}(s) G_{d}(s) G_{r}(s)$, with an unitary feedback. In this way, the stability conditions of the proposed auxiliary closed-loop system are equivalent to the required stability conditions for the characteristic equation (36).

The Nyquist stability criterion states that for a given open-loop system $\bar{Q}(s)$, there is a stabilizing proportional control $k$ such that the closed-loop system is stable if and only if $0=N+P$, where $P$ is the number of unstable roots in $\bar{Q}(s)$ and $N$ is the number of clockwise round trips to the point $(-1$, $0 j$ ) in the open-loop $(\bar{Q}(s))$ Nyquist diagram. It can be seen that for our particular case $Q(s)=\bar{C}(s) G_{d}(s) G_{r}(s)$,

$P=2$ and therefore $N$ should satisfy $N=-2$, i.e., in order to achieve closed-loop stability the Nyquist plot should encircle twice the critical point $(-1,0 j)$ in counter-clockwise direction. Therefore, the selection of the derivative should be proposed to obtain a Nyquist trajectory such that the closedloop system is stable, in this way a desirable behaviour of the trajectory should be as it is shown in Figure 5 or Figure 6.

For the sake of simplicity on the frequency domain analysis, let use an alternative representation of the PD controller given by,

$$
\bar{C}(s)=k_{p}\left(k_{d} s-1\right),
$$

with $k_{p}, k_{d}>0$. Notice that (37) is a non-minimum phase controller. The selection of this controller structure allows to us to find the best stability conditions in terms of the delay value.

Let us assume that the condition (34) holds, it could be selected a $k_{d}$ gain for the PD controller such that,

$$
\bar{k}_{d}=\frac{1}{a}+\frac{1}{c}-\sum_{i=1}^{m} \frac{1}{b_{i}}-\sum_{i=1}^{n} \frac{1}{d_{i}}-\theta+\varepsilon,
$$

with $\varepsilon$ being a sufficient small positive constant.

In order to satisfy the Nyquist stability criteria a frequency domain analysis of the system $Q(j \omega)=\bar{C}(j \omega) G_{d}(j \omega) G_{r}(j \omega)$ is required and performed in what follows. Thus, the phase equation of the system is,

$$
\Phi_{Q}(\omega)=\arctan \left(\frac{\omega}{a}\right)+\arctan \left(\frac{\omega}{c}\right)-\sum_{i=1}^{m} \arctan \left(\frac{\omega}{b_{i}}\right)-\sum_{i=1}^{n} \arctan \left(\frac{\omega}{d_{i}}\right)-\arctan \left(\bar{k}_{d} \omega\right)-\omega \theta-\pi
$$




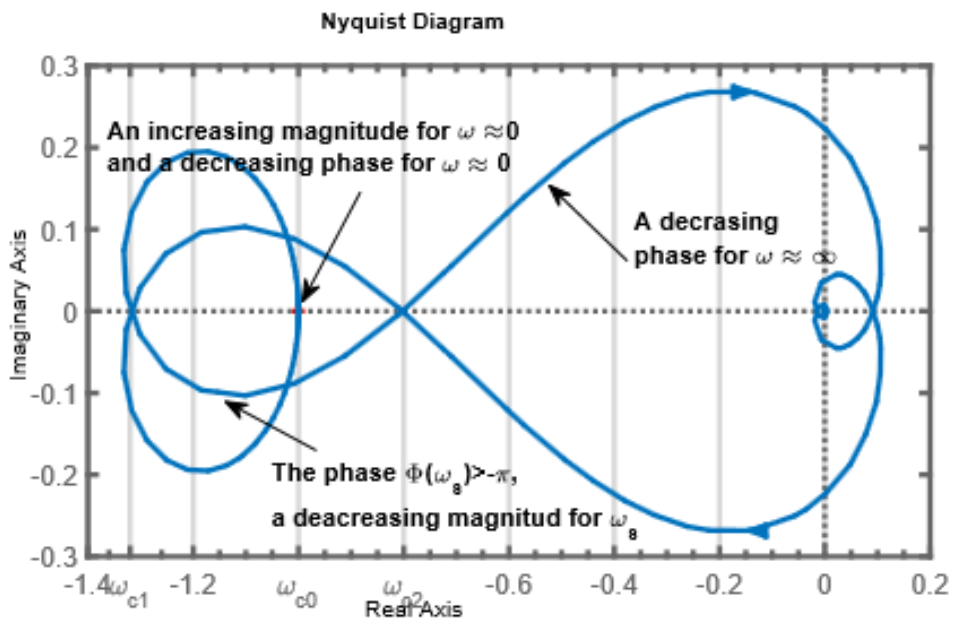

Figure 5: Desired stable Nyquist trajectory of the system with two open-loop unstable poles.

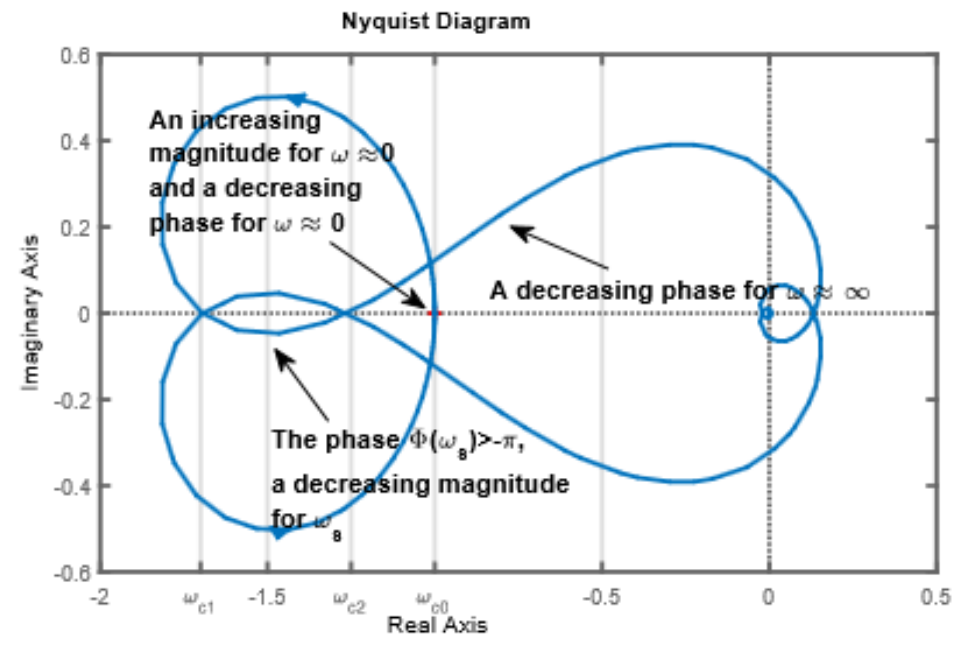

Figure 6: Desired stable Nyquist trajectory of the system with two open-loop unstable poles.

It can be seen that the phase begins in $\Phi_{Q}(0)=-\pi$ and its trajectory follows a negative direction, this could be demonstrated considering the derivative of the phase,

$$
\frac{d \Phi_{Q}(\omega)}{d \omega}=\frac{a}{\omega^{2}+a^{2}}+\frac{c}{\omega^{2}+c^{2}}-\sum_{i=1}^{m} \frac{b_{i}}{\omega^{2}+b_{i}{ }^{2}}-\sum_{i=1}^{n} \frac{d_{i}}{\omega^{2}+d_{i}{ }^{2}}-\frac{\bar{k}_{d}}{\bar{k}^{2}{ }_{d} \omega^{2}+1}-\theta,
$$

for frequencies in the neighborhood of $\omega \approx 0$, 


$$
\left.\frac{d \Phi_{Q}(\omega)}{d \omega}\right|_{\omega \approx 0}=\frac{1}{a}+\frac{1}{c}-\sum_{i=1}^{m} \frac{1}{b_{i}}-\sum_{i=1}^{n} \frac{1}{d_{i}}-\bar{k}_{d}-\theta<0 .
$$

From (41), the starting angle of the system depends on the value of $\epsilon$. It can be seen that for an $\varepsilon \rightarrow 0$, the initial direction of the phase is slightly negative, which yields a crossover frequency $\omega_{c_{1}} \rightarrow 0$, where $\omega_{c_{1}}$ is the first frequency $\omega_{c 1}>0$ such that $\Phi_{Q}\left(\omega_{c_{1}}\right)=-\pi$. Moreover, there is a frequency $\omega_{s}=\sqrt{a c}$ such that $\Phi_{Q}\left(\omega_{s}\right)>-\pi$, this can be found numerically.

Additionally, analyzing the phase derivative for high frequencies, namely $\omega \approx \infty$, we can see that its trajectory is decreasing for high frequencies. In order to complete the analysis let us consider the magnitude equation given by,

$$
M_{Q}(\omega)=K \sqrt{\frac{\left(\bar{k}^{2}{ }_{d} \omega^{2}+1\right)}{\left(\frac{\omega^{2}}{a^{2}}+1\right)\left(\frac{\omega^{2}}{c^{2}}+1\right) \prod_{i=1}^{m}\left(\frac{\omega^{2}}{b_{i}^{2}}+1\right) \prod_{i=1}^{n}\left(\frac{\omega^{2}}{d_{i}{ }^{2}}+1\right)}},
$$

with $K=\frac{\bar{\alpha} k_{p}}{a c} \prod_{i=1}^{m} \frac{1}{b_{i}} \prod_{i=1}^{n} \frac{1}{d_{i}}$ and $\bar{\alpha}=\alpha \beta$.

For small frequencies, the magnitude has a positive direction, which is easily concluded from:

$$
\left.\frac{d M_{Q}(\omega)}{d \omega}\right|_{\omega \approx 0} \approx \bar{k}_{d}^{2}-\frac{1}{a^{2}}-\frac{1}{c^{2}}-\sum_{i=1}^{m} \frac{1}{b_{i}^{2}}-\sum_{i=1}^{n} \frac{1}{d_{i}^{2}}>0
$$

Since the open-loop system $Q(j \omega)$ is strictly proper, there is a decreasing magnitude for large frequencies. As it can be observed, the selection of $\bar{k}_{d}$ results in a system with a positive gain margin, for a positive crossover frequency $\omega_{c_{1}} \rightarrow 0$, and a positive phase margin occurring at $\omega_{m}>0$. Then, it can be concluded that the system $G_{d}(s) G_{r}(s)$ can be stabilized with a PD controller (37) choosing a derivative gain according to (38); and equivalently the recycling system (1) can be stabilized with a PD controller (32) in the control loop shown in Figure 2.

Necessity. Taking into account Lemma 3, the stability of the closed-loop system with a PD controller requires that the polynomial:

$$
\mathrm{H}_{Q}(\mathrm{~s})=\gamma_{0}+\gamma_{1} \mathrm{~s}+\gamma_{2} \mathrm{~s}^{2}+\cdots+\gamma_{n+1} \mathrm{~s}^{n+1}+\gamma_{n+2} \mathrm{~s}^{n}
$$

has all its zeros in the open left half plane, since the higher order coefficient:

$$
\gamma_{m+n+2}=\frac{\theta^{2}}{a c} \prod_{i=1}^{m} \frac{1}{b_{i}} \prod_{i=1}^{n} \frac{1}{d_{i}}
$$

is a positive constant, a necessary condition for the stabilizability is that the independent term

$$
\gamma_{0}=\theta^{2}+\left(\sum_{i=1}^{m} \frac{1}{b_{i}}+\sum_{i=1}^{n} \frac{1}{d_{i}}-\frac{1}{a}-\frac{1}{c}\right) \theta+\frac{1}{a c}-\left(\frac{1}{a}+\frac{1}{c}\right)\left(\sum_{i=1}^{m} \frac{1}{b_{i}}+\sum_{i=1}^{n} \frac{1}{d_{i}}\right)+\sum_{i=1}^{m-1} \sum_{j>i}^{m} \frac{1}{b_{i} b_{j}}+\sum_{i=1}^{n-1} \sum_{j>i}^{n} \frac{1}{d_{i} d_{j}},
$$

is positive. Solving for the delay term we have:

$$
\theta<\frac{1}{a}+\frac{1}{c}-\sum_{i=1}^{m} \frac{1}{b_{i}}-\sum_{i=1}^{n} \frac{1}{d_{i}}-\sqrt{\frac{1}{a^{2}}+\frac{1}{c^{2}}+\sum_{i=1}^{m} \frac{1}{b_{i}^{2}}+\sum_{i=1}^{n} \frac{1}{d_{i}^{2}}} .
$$


Remark 1 An adequate choice of the derivative gain $k_{d}$ assures a Nyquist path with two encirclements in counter-clockwise direction, namely the system can be stabilized. In order to obtain a closed-loop stable system, the proportional gain $k_{p}$ should be selected such that:

$$
\frac{a c \prod_{i=1}^{m} b_{i} \prod_{i=1}^{n} d_{i}}{\bar{\alpha} F\left(\omega_{c 1}\right)}<k_{p}<\frac{a c \prod_{i=1}^{m} b_{i} \prod_{i=1}^{n} d_{i}}{\bar{\alpha} F\left(\omega_{c q}\right)},
$$

where $q=0$ for $F\left(\omega_{c 2}\right)<F\left(\omega_{c 0}\right)<F\left(\omega_{c 1}\right), q=2$ for $F\left(\omega_{c 0}\right)<F\left(\omega_{c 2}\right)<F\left(\omega_{c 1}\right)$,

$$
F\left(\omega_{c^{*}}\right)=\sqrt{\left.\frac{\left(\bar{k}_{d}^{2} \omega^{2}{ }^{2}+1\right)}{a^{2}{ }^{2}}+1\right)\left(\frac{\omega^{2} c^{*}}{c^{2}}+1\right) \prod_{i=1}^{m}\left(\frac{\omega^{2} c^{*}}{b_{i}{ }^{2}}+1\right) \prod_{i=1}^{n}\left(\frac{\omega^{2} c^{*}}{d_{i}{ }^{2}}+1\right)},
$$

$\omega_{c 0}=0, \omega_{c_{1}}$ and $\omega_{c 2}$ are denominated the first and second positive crossover frequency such that $\Phi_{Q}\left(\omega_{c_{1}}\right)=-\pi$, respectively.

The frequency domain analysis used in the proof of Theorem 2 can be applied to the case of the PID controller obtaining the same stability condition. In this way, the following result is stated.

Corollary 1 Consider the feedback control shown in Figure 2. Then, the delayed recycling system given by (1) can be stabilized with a PID controller given by,

$$
C(s)=K_{p}+\frac{K_{I}}{s}+K_{D} s
$$

or

$$
C(s)=k_{p}\left(k_{d} s-1+\frac{k_{i}}{s}\right)+1,
$$

(with $k_{p}, k_{i}, k_{d}>0$ ) if and only if the condition (34) is satisfied.

Remark 2 An adequate selection of the derivative gain $k_{d}$ and the integral term $k_{i}$ in the PID controller given by (46) assures a Nyquist path with two counter-clockwise direction, i.e., the closed-loop stability warranty. In order to obtain a closed-loop stable system, the proportional gain $k_{p}$ should be selected such that,

$$
\frac{a c \prod_{i=1}^{m} b_{i} \prod_{i=1}^{n} d_{i}}{\bar{\alpha} \gamma\left(\omega_{c 1}\right)}<k_{p}<\frac{a c \prod_{i=1}^{m} b_{i} \prod_{i=1}^{n} d_{i}}{\bar{\alpha} \gamma\left(\omega_{c r}\right)},
$$

where $r=2$ for $\gamma\left(\omega_{c 3}\right)<\gamma\left(\omega_{c 1}\right)<\gamma\left(\omega_{c 2}\right), r=3$ for $\gamma\left(\omega_{c 1}\right)<\gamma\left(\omega_{c 3}\right)<\gamma\left(\omega_{c 2}\right)$,

$$
\gamma\left(\omega_{c^{*}}\right)=\sqrt{\frac{\left(-\omega^{2} c^{*} k_{d}+k_{i}\right)^{2}+\omega^{2} c^{*}}{\omega^{2}{ }_{c^{*}}\left(\frac{\omega^{2} c^{*}}{a^{2}}+1\right)\left(\frac{\omega^{2} c^{*}}{c^{2}}+1\right) \prod_{i=1}^{m}\left(\frac{\omega^{2} c^{*}}{b_{i}{ }^{2}}+1\right) \prod_{i=1}^{n}\left(\frac{\omega^{2} c^{*}}{d_{i}{ }^{2}}+1\right)}} .
$$

where $\omega_{c 1}, \omega_{c 2}, \omega_{c 3}>0$ are denominated the first and second, third crossover frequencies respectively, such 
that $\Phi(\omega)=-\pi$, where

$$
\Phi(\omega)=\arctan \left(\frac{\omega}{a}\right)+\arctan \left(\frac{\omega}{c}\right)-\sum_{i=1}^{m} \arctan \left(\frac{\omega}{b_{i}}\right)-\sum_{i=1}^{n} \arctan \left(\frac{\omega}{d_{i}}\right)-\arctan \left(\bar{k}_{d} \omega-\frac{k_{i}}{\omega}\right)-\omega \theta-\pi
$$

A simple procedure to determine the PD/PID controller parameters is proposed.

\section{Procedure 2}

1. Identify the plant parameters $a, c, b i, d i, \bar{\alpha}, \theta$.

2. If the stability condition (34) holds, continue with step 3; otherwise, a PD or PID controller can not stabilize the recycling delayed process (1) in the control scheme shown in Figure 2.

3. Compute the minimum stabilizing derivative gain $k d$ for the PD/PID controller (given by (33)/(46)) according to (38).

4. For the controller (33)/(46), search for a maximum $k_{d}$ such that the phase equation $\Phi_{Q}\left(\omega_{c_{1}}\right)=-\pi$ (given by (39)), for some $\omega_{c 1}>0 \omega_{c_{1}}>0$ where $\omega_{m}>\omega_{c_{1}}>0$ and $\omega_{m}$ is the frequency such that $M\left(\omega_{m}\right)=1$.

5. If a PD controller is required, select a stabilizing kd from the obtained interval in Steps 3-4 and compute the stabilizing $\mathrm{kp}$ interval using (43).

6. If a PID controller is required, select a stabilizing $k_{d}$ from the obtained interval in Steps 3-4 and obtain the maximal $k_{i}\left(k_{i_{\text {max }}}\right)$, starting from $k_{i}=0$ such that $\Phi(\omega)>-\pi(\Phi(\omega)$ given by (49)) for some $\omega>0$. Then, select a $k_{i}$ into the interval $0<k_{i}<k_{i_{\text {max }}}$ and with the selected $k_{d}$ and $k_{i}$ compute the stabilizing $k_{p}$ interval using (47).

Remark 3 Notice that a particular case of the condition(34) in Theorem 2 can be obtained for the case of two unstable poles, i.e.

$$
\theta<\frac{1}{a}+\frac{1}{c}-\sqrt{\frac{1}{a^{2}}+\frac{1}{c^{2}}}
$$

In this way, since it is assumed $\theta>0$ implies $\frac{1}{a}+\frac{1}{c}-\sqrt{\frac{1}{a^{2}}+\frac{1}{c^{2}}}>0$ which from a graphical viewpoint satisfies the properties of a right triangle as well as the inequality triangle $\left(\frac{1}{a}+\frac{1}{c}>\sqrt{\frac{1}{a^{2}}+\frac{1}{c^{2}}}\right)$, see Figure 7 .

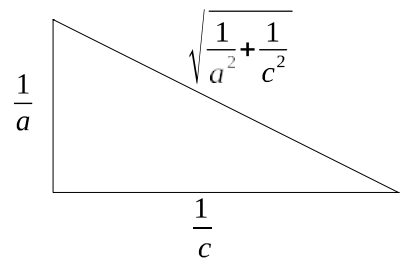

Figure 7: Graphical interpretation of condition (50). 


\subsection{Proposed observer based control}

To implement the results presented in Sections 4.1 and 4.2 through an observer based controller, Figure 8 shows the complete scheme and the following result is presented in order to assure the stability condition of the whole proposed strategy.

Corollary 2 Consider the observer-based control depicted in Figure 8. Then, there exists a controller, C(s), $\mathrm{PD} / \mathrm{PID}$ such that the closed-loop system is stable if and only if,

$$
\theta<\frac{1}{a}+\frac{1}{c}-\sum_{i=1}^{m} \frac{1}{b_{i}}-\sum_{i=1}^{n} \frac{1}{d_{i}}-\sqrt{\frac{1}{a^{2}}+\frac{1}{c^{2}}+\sum_{i=1}^{m} \frac{1}{b_{i}^{2}}+\sum_{i=1}^{n} \frac{1}{d_{i}^{2}}}
$$

with $\theta=\tau_{1}+\tau_{2}$

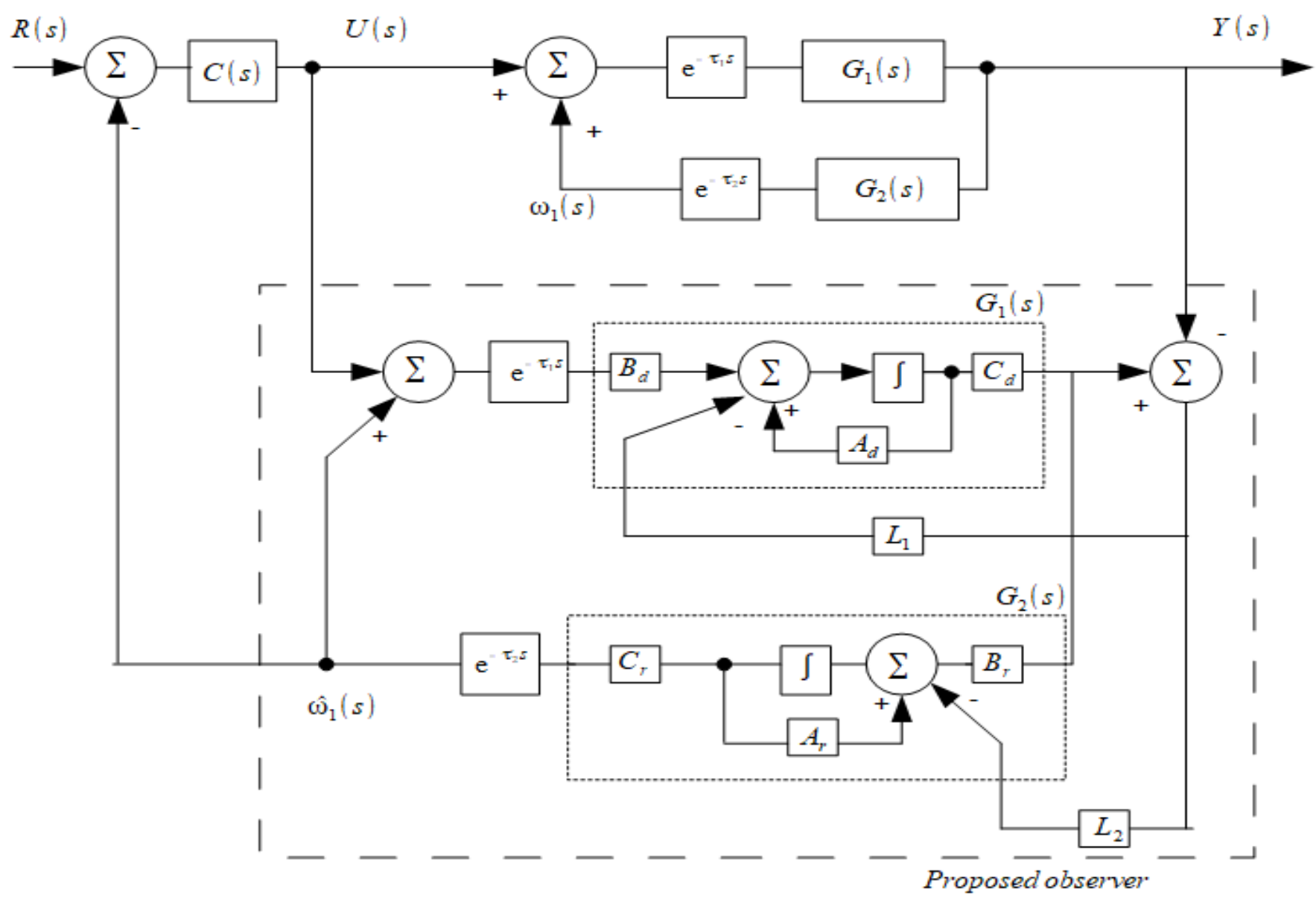

Figure 8: Proposed observer based control schema.

Proof. Consider the observer scheme shown in Figure 8. From Theorem 1, an adequate estimation

$\widehat{\omega}_{1}(s)$, of the signal $\omega_{1}(s)$ is assured if and only if 


$$
\theta<\frac{1}{c}-\sum_{i=1}^{n} \frac{1}{d_{i}}-\sum_{i=1}^{m+1} \frac{1}{z_{i}}
$$

for $\theta=\tau_{1}+\tau_{2}$. On the other hand, by Theorem 1 and Corollary 1 it is possible to find a controller $\mathrm{PD} / \mathrm{PID}$ such that the closed-loop system is stable if and only if,

$$
\theta<\frac{1}{a}+\frac{1}{c}-\sum_{i=1}^{m} \frac{1}{b_{i}}-\sum_{i=1}^{n} \frac{1}{d_{i}}-\sqrt{\frac{1}{a^{2}}+\frac{1}{c^{2}}+\sum_{i=1}^{m} \frac{1}{b_{i}^{2}}+\sum_{i=1}^{n} \frac{1}{d_{i}^{2}}}
$$

with $\theta=\tau_{1}+\tau_{2}$. Then we can conclude that the closed-loop system of Figure 8 is stable if and only if the most restrictive condition between the conditions (52) and (53) is satisfied, i.e., the condition (53).

The proposed control strategy can be also extended to a class of systems that considers unstable dynamic at direct path but only stable dynamics at the recycling path. The following result establish how to apply the corresponding extension.

\section{Corollary 3. Consider the observer-based controldepicted in Figure 8 with}

$$
\begin{aligned}
& G_{d}(s)=G_{1}(s) e^{-\tau_{1} s}=\frac{\alpha}{D_{1}(s)} e^{-\tau_{1} s} \\
& G_{r}(s)=G_{2}(s) e^{-\tau_{2} s}=\frac{\beta}{\bar{D}_{2}(s)} e^{-\tau_{2} s}
\end{aligned}
$$

where $D_{1}(s)=(s-a)\left(s+b_{1}\right) \ldots\left(s+b_{m}\right)\left(s^{2}+2 \xi \omega_{n}+\omega_{n}^{2}\right)$ and $\bar{D}_{2}(s)=\left(s+d_{1}\right)\left(s+d_{2}\right) \ldots\left(s+d_{n}\right)$, where stable poles could be complex conjugate. Then, there exists a controller, C(s), PD/PID such that the closed-loop system is stable if and only if,

$$
\theta<\frac{1}{a}-\sum_{i=1}^{m} \frac{1}{b_{i}}-\sum_{i=1}^{n} \frac{1}{d_{i}}-2 \frac{\xi}{\omega_{n}}-\sqrt{\frac{1}{a^{2}}+\sum_{i=1}^{m} \frac{1}{b_{i}^{2}}+\sum_{i=1}^{n} \frac{1}{d_{i}^{2}}+2 \frac{\xi}{\omega_{n}^{2}}}
$$

Proof. Consider the observer scheme shown in Figure 8. An adequate estimation $\widehat{\omega}_{1}(s)$, of the signal $\omega_{1}(s)$ is always assured since the parameter $L_{2}$ can be performed via a Nyquist plot.

On the other hand, by Theorem 2 in [17] it is possible to find a controller PD/PID such that the closed-loop system is stable if and only if,

$$
\theta<\frac{1}{a}-\sum_{i=1}^{m} \frac{1}{b_{i}}-\sum_{i=1}^{n} \frac{1}{d_{i}}-2 \frac{\xi}{\omega_{n}}-\sqrt{\frac{1}{a^{2}}+\sum_{i=1}^{m} \frac{1}{b_{i}^{2}}+\sum_{i=1}^{n} \frac{1}{d_{i}^{2}}+2 \frac{\xi}{\omega_{n}^{2}}}
$$

with $\theta=\tau_{1}+\tau_{2}$. Then we can conclude that the closed-loop system of Figure 8 is stable if and only if the most restrictive condition between the convergence condition and controller condition (55) is satisfied. In such case, we have (55). 


\section{Numerical Results}

Let us start with a theoretical and academic example that illustrstes all the potentiallity of the results here exposed. Later, a more realistic example borrowed from the literature is presented.

Example 1. Consider a recycling process with the transfer function

$$
\begin{aligned}
& G_{d}(s)=\frac{0.5}{(s-0.4)(s+12)(s+20)} e^{-0.2 s,} \\
& G_{r}(s)=\frac{0.5}{(s-0.8)(s+9)(s+11)} e^{-0.1 s,}
\end{aligned}
$$

First, the observer design is regarded, hence the Procedure 1 is followed. The existence of the observer strategy is assured due to the condition (24) in Theorem 1 is satisfied, namely $\theta<1.0480$. The relocated poles of $G_{1}$ are proposed at $\{-8-9-10\}$ i.e., $z_{1}=8, z_{2}=9$ and $z_{3}=10$. Taking into account the desired relocated poles previously proposed, (29) is solved to $L_{1}$, obtaining $L_{1}=\left[\begin{array}{lll}821.18 & 104.96 & -4.6\end{array}\right]^{T}$. Using the Step 3 of Procedure 1 the interval of the observer parameter $\bar{l}_{2-(1)}$ is $57025<\bar{L}_{2-(1)}<104150$. Secondly, the design of the controller $C(\mathrm{~s})$ is derived. To this end, Procedure 2 is considered to design a PD controller. Figure 9 shows the complete stabilizing region $k_{p}-k_{d}$ for the controller given by (33). In order to illustrate the behaviour of the proposed control strategy, some simulations are performed, Figure 10 shows the output estimation error defined as $e_{y}(t)=y(t)-y(t)$ when initial conditions different from zero are considered in the states of the plant, i.e., [0.01,0.01,0.01]. The output performance is presented in Figure 11 under different scenarios: initial conditions are equal to zero, initial conditions are $[0.1,0.1,0.1]$ and an uncertainty in the time-delay $\tau_{2}$ of $+20 \%$ is present in the plant. For the simulation experiment $\bar{l}_{2-(1)}=63025, k_{p}=7000$ and $k_{d}=4$ are used.

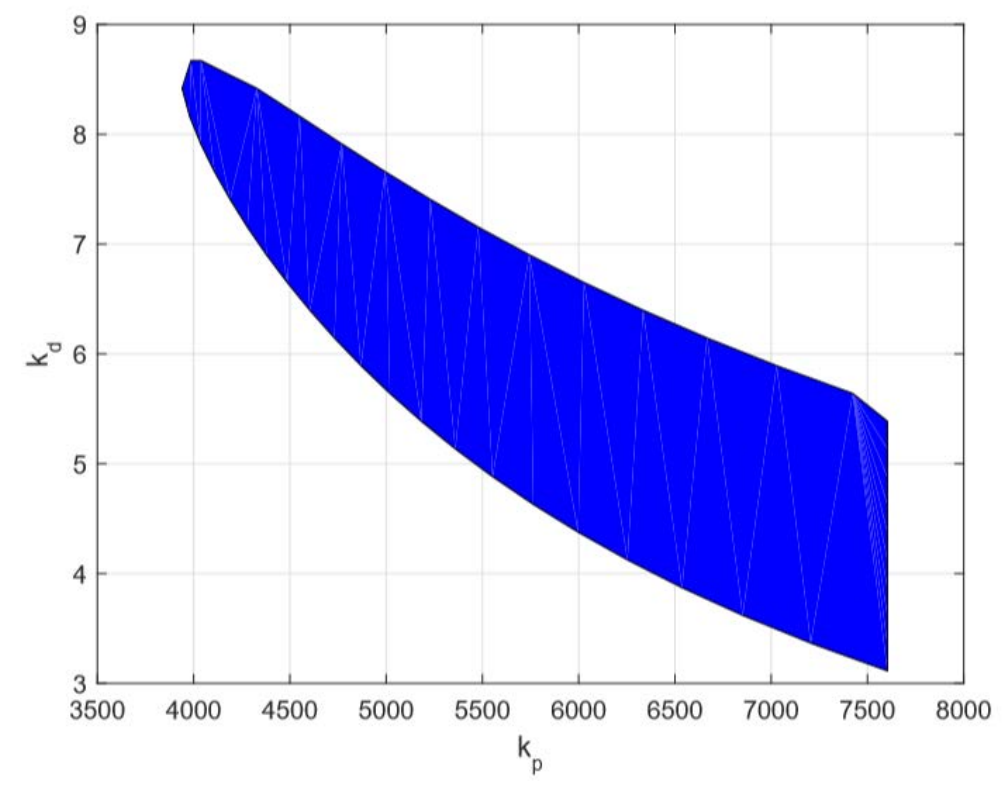

Figure 9: Stabilizing $k_{p}-k_{d}$ region for the PD controller (33) for Example 1. 


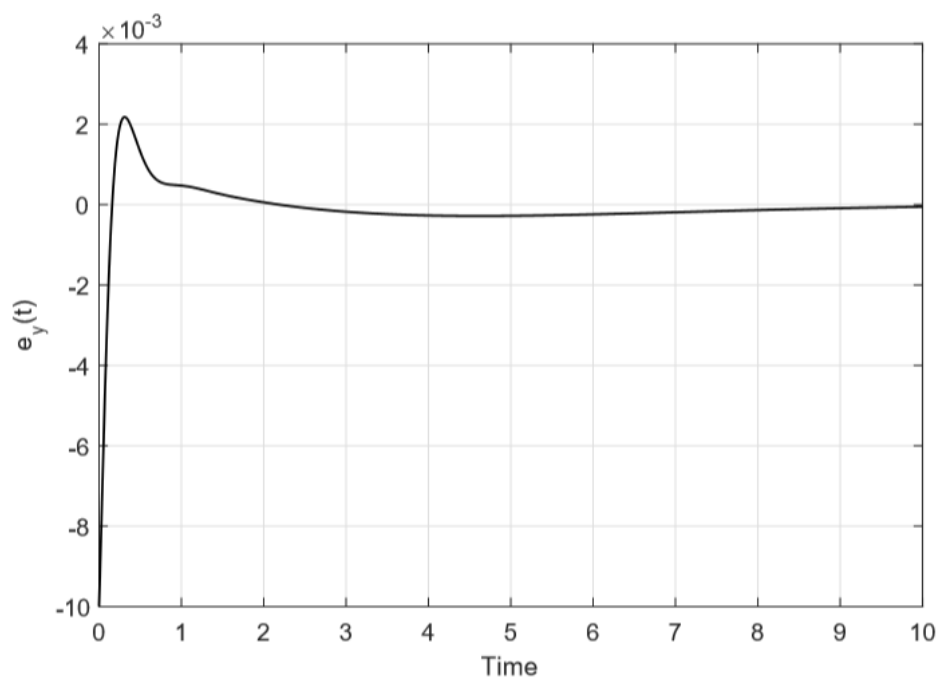

Figure 10: Output estimation error, $e_{y}(t)=\hat{y}(t)-y(t)$, for Example 1.

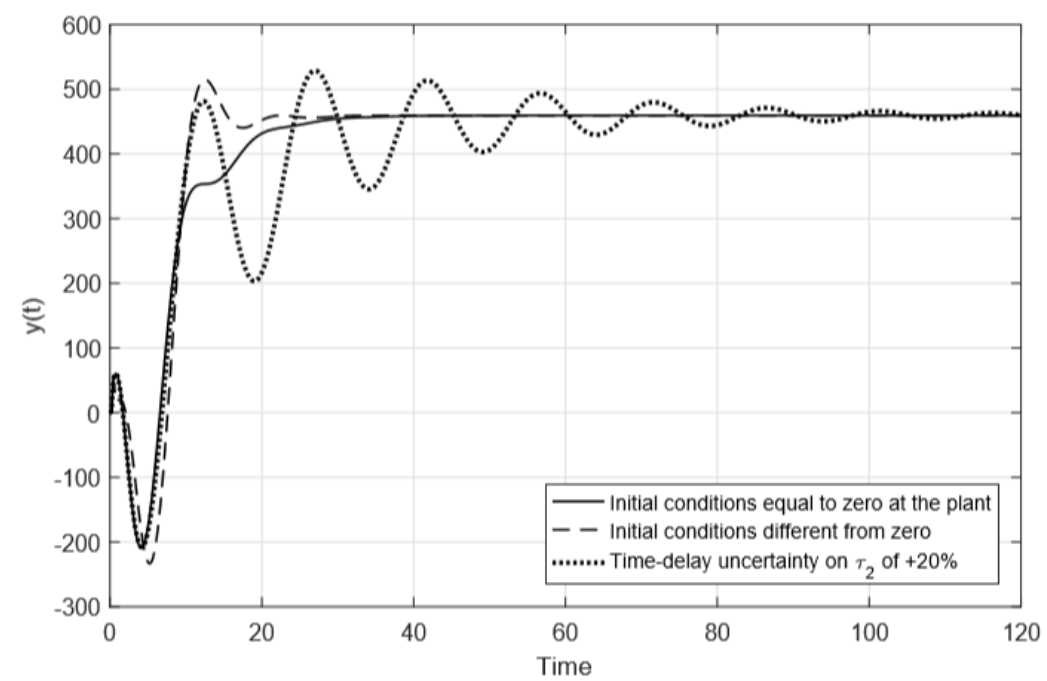

Figure 11: Output response for Example 1 using a PD controller.

Figure 11 shows the closed-loop of the controlled system. It can be seen that the transitory behaviour of the output presents an initial undershoot and oscillations in the opposite direction with respect to the final stationary response. It is important to recall that we are dealing with a very complex delayed system with two unstable poles. The interconnection among the plant, the observer as well as the controller induces such characteristic response. In a practical situation, the inverse response could be bounded to zero, it depends on the nature of the variable. It can also be seen that if the delay size is not well characterized (for instance $+20 \%$ of error), the stability is preserved but oscillations are present in the output signal.

Now, if a PID controller is considered to stabilize the plant given by (54), the stabilizing parameters plane $k_{d}-k_{i}$ is shown in Figure 12, which is obtained using Step 6 from Procedure 1. Notice that for each point $k_{d}-k_{i}$ into the stabilizing region, a $k_{p}$ interval should be computed using (47). In this way some stabilizing triplet $k_{p}-k_{i}-k_{d}$ are presented in Figure 13. 


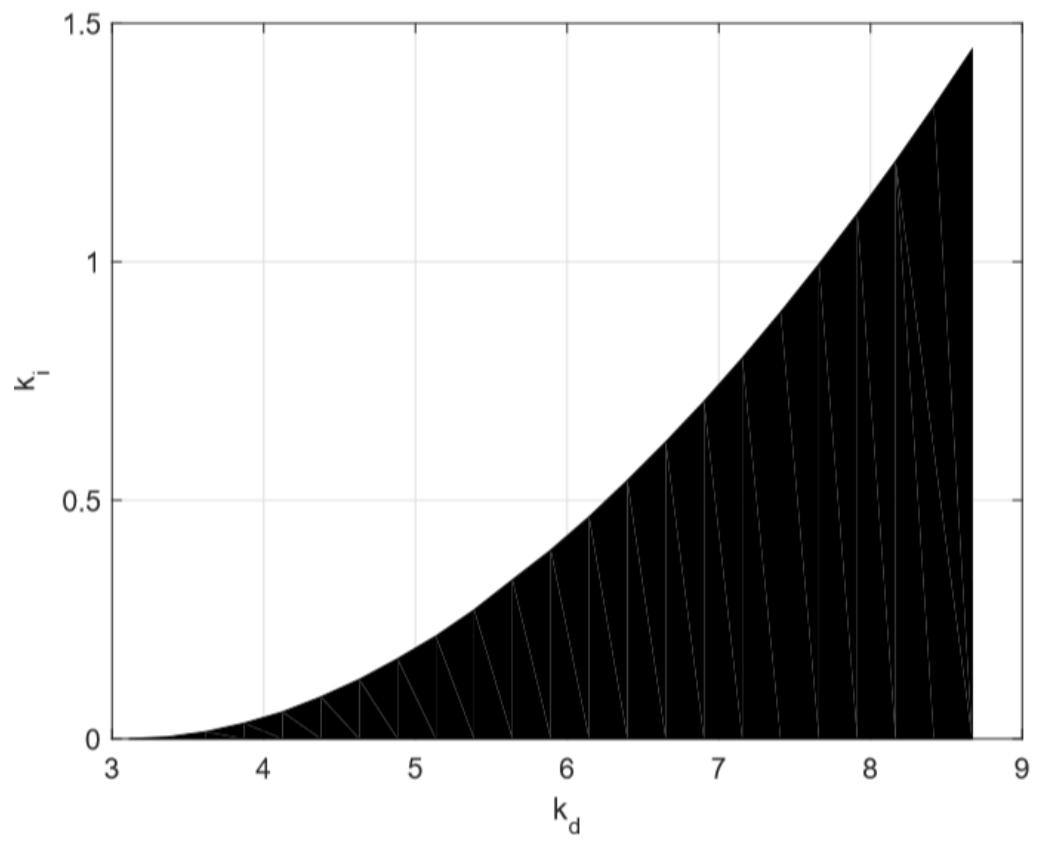

Figure 12: Stabilizing $k_{d}-k_{i}$ region for the PID controller (33) for Example 1.

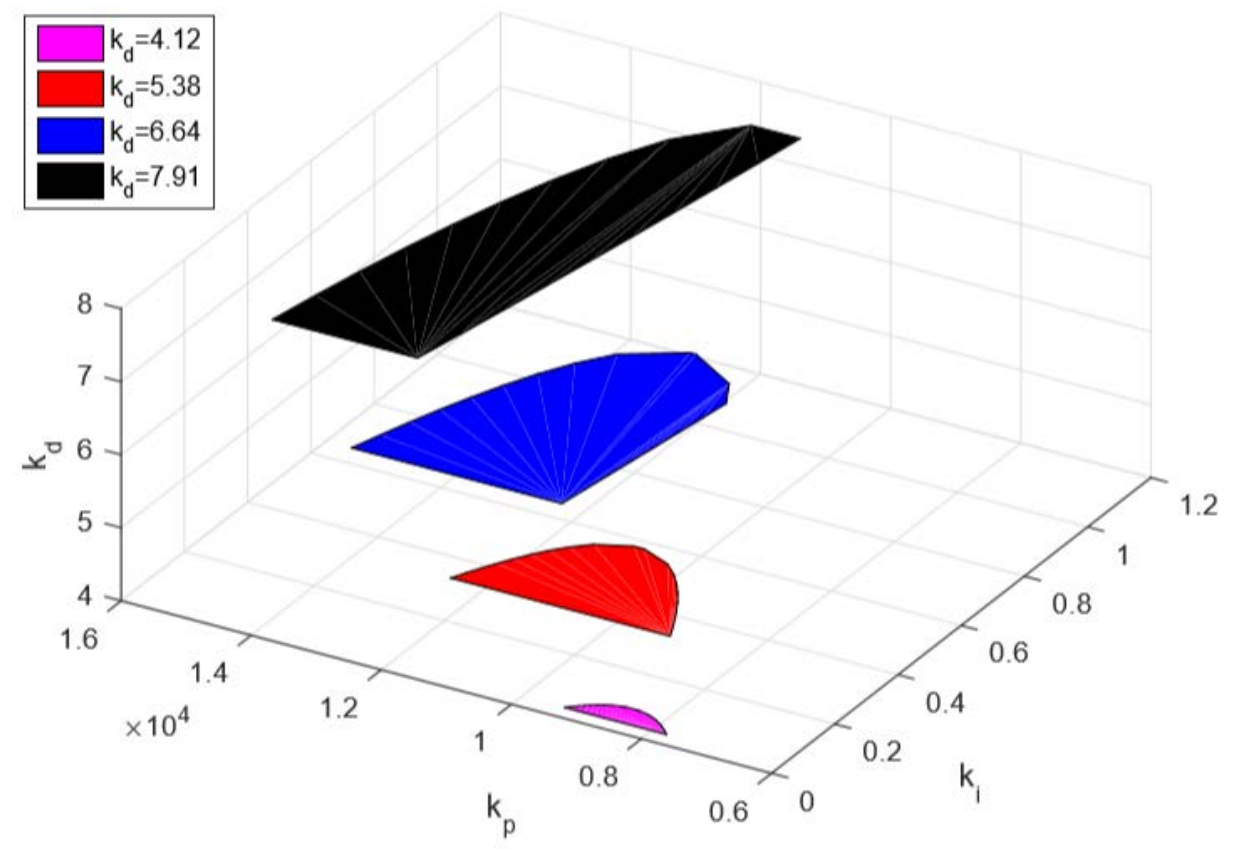

Figure 13: Stabilizing $k_{p}-k_{d}-k_{i}$ region for the PID controller (33) for Example 1. 
Example 2. Consider the reactor-separator recycle plant presented in [21], time delays at direct and recycling paths are included. Thus an open-loop state space representation is,

$$
\begin{gathered}
x(t)=\bar{A} x(t)+\bar{A}_{1} x\left(t-\tau_{1}\right)+\bar{A}_{2} x\left(t-\tau_{2}\right)+\bar{B} u(t), \\
y(t)=\bar{C}_{1} x\left(t-\tau_{1}\right),
\end{gathered}
$$

where,

$$
\begin{aligned}
& x(t)=\left[\begin{array}{ll}
x_{T}(t) & x_{C}(t)
\end{array}\right]^{T}, \bar{A}=\left[\begin{array}{cc}
\frac{F}{V}(1-\lambda)-\frac{F}{V}-\frac{U A}{V \rho c_{p}}+\frac{(-\Delta H)}{\rho c_{p}} C_{A S} k_{p s} & 0 \\
0 & \frac{F}{V}(1-\lambda)-\frac{F}{V}-k_{s}
\end{array}\right] . \\
& \bar{A}_{1}=\left[\begin{array}{cc}
0 & 0 \\
-C_{A S} k_{p s} & 0
\end{array}\right], \bar{A}_{2}=\left[\begin{array}{cc}
0 & \frac{(-\Delta H)}{\rho c_{p}} k_{s} \\
0 & 0
\end{array}\right], B=\left[\begin{array}{c}
\frac{U A}{V \rho c_{p}} \\
0
\end{array}\right], k_{s}=k_{0} \exp \left(-E_{a} / R T_{s}\right) \text { and } k_{p s}=k_{s} \exp \left(E_{a} / R T_{s}^{2}\right) .
\end{aligned}
$$

\begin{tabular}{|l|l|}
\hline Operating volumen $(V)$ & $500 \mathrm{ft}^{3}$ \\
\hline Operating flowrate $(F)$ & $2000 \mathrm{ft}^{3} / \mathrm{hr}$ \\
\hline Reactor diameter $\left(D_{r}\right)$ & $7.5 \mathrm{ft}$ \\
\hline Overall heat-transfer coefficient $(U)$ & $492.3192 \mathrm{Btu} /\left(\mathrm{hrft}^{2}{ }^{\circ} \mathrm{R}\right)$ \\
\hline Heat transfer área through reactor wall $(A)$ & $47.1238 \mathrm{ft}^{2}$ \\
\hline Preexponential factor $\left(k_{0}\right)$ & $3.96 \times 10^{12} \mathrm{hr}^{-1}$ \\
\hline Activation energy $\left(E_{a}\right)$ & $32400 \mathrm{Btu} / \mathrm{lmol}$ \\
\hline Ideal gas constant $(R)$ & $1.987 \mathrm{Btu} / \mathrm{lbmol}{ }^{\circ} \mathrm{R}$ \\
\hline Heat of reaction $(-\Delta H)$ & $39000 \mathrm{Btu} / \mathrm{lbmolPO}$ \\
\hline Density of coolant $(\rho)$ & $53.25 \mathrm{lb} / \mathrm{ft}^{3}$ \\
\hline Heat capacity of coolant $\left(C_{\rho}\right)$ & $1 \mathrm{Btu} /\left(\mathrm{lb} \mathrm{b}^{\circ}\right)$ \\
\hline Operating concentration $\left(C_{A s}\right)$ & $0.066 \mathrm{lbmol} / \mathrm{ft}^{3}$ \\
\hline Operating temperatura $\left(T_{s}\right)$ & $560.77^{\circ} \mathrm{R}$ \\
\hline Direct path time delay $\left(\tau_{1}\right)$ & $0.15 \mathrm{hr}$ \\
\hline Recycling path time delay $\left(\tau_{2}\right)$ & $0.18 \mathrm{hr}$ \\
\hline Recirculation coefficient $(\lambda)$ & 0.2 \\
\hline
\end{tabular}

Table 1. Parameters of system in Example 2.

The parameter values are listed in Table 1. $x_{T}(t)$ is the reactor temperature $(T)$ and $x_{C}(t)$ is the component concentration $\left(C_{A}\right)$. The input variable is the jacket reactor temperature $\left(T_{j}\right) . \tau_{1}$ is the time-delay due to the concentration and $\tau_{2}$ is the time delay derived from matherial transport. Output matrix, $\bar{C}_{1}=\left[\begin{array}{ll}0 & 1\end{array}\right]$, is assumed. Thus, transfer functions are obtained as:

$$
\begin{gathered}
G_{d}(s)=\frac{-2.778 \times 10^{-3}}{(s-0.6643)(s+1.732)} e^{-0.15 s}, \\
G_{r}(s)=7.8323 \times 10^{2} e^{-0.18 s},
\end{gathered}
$$

Taking into account Corollary 3, the condition (5) is satisfied. From Step 2 of Procedure 1, it is proposed to relocate the 


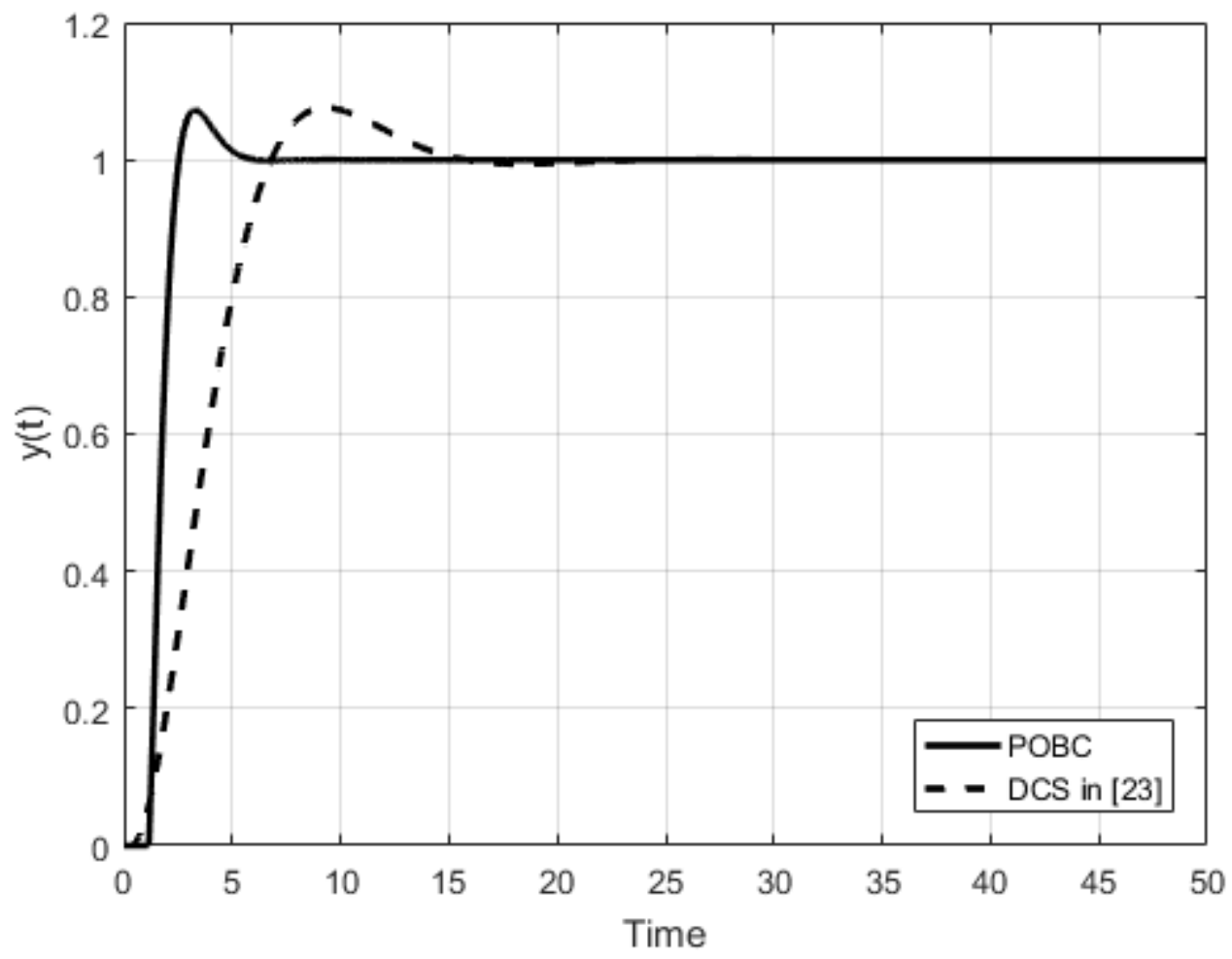

Figure 14: Nominal performance comparison of Example 2.

poles of direct path such that $z_{1}=1, z_{2}=2$. This, gives as result the oberver paremeter $L_{1}=\left[\begin{array}{ll}4.4341 & 1.9323\end{array}\right]^{T}$. Then, from a Nyquist stability analysis the observer parameter $-3035<L_{2}<0$ is obtained. For the experiment $L_{2}=-10$ is used. Finally, following the methodology in [17], the used PD controller is given as,

$$
C(s)=K_{p}+K_{D} s,
$$

with $K_{p}=0.1$ and $K_{D}=0.55$. In order to improve the performance a set point filter is used,

$$
f_{s p}(s)=\frac{0.55 s+1.1}{0.55+0.1} .
$$

A comparison with a recent work related to control of recycling plant proposed in [23] is performed. In [23], a delayed control strategy (DCS) is used with $C(s)=290 e^{-0.18 s}$. Step reference adjustment were used in order to obtain unitary state staionary value. Figure 14 shows a comparison of the output performance under nominal conditions, the proposed observer-based control in this manuscript is denoted as POBC. Closedloop robustness with respect to parametric modelling uncertainties into system (56a)-(56b) is illustrated in what follows. Consider the recycling system under additive bounded uncertainties, given by,

$$
\begin{gathered}
G_{d}(s)=\frac{\alpha}{(s-\bar{a})\left(s+b_{1}\right)} e^{-\overline{\tau_{1}} s} \\
G_{r}(s)=\beta e^{-\overline{\bar{z}_{2}} s}
\end{gathered}
$$

where additive uncertainties are defined as $\bar{a}=a+\Delta_{a}, \overline{\tau_{1}}=\tau_{1}+\Delta_{\tau_{1}}$ and $\overline{\tau_{2}}=\tau_{2}+\Delta_{\tau_{2}}$. In this way, considering $a=0.6643$ and $\Delta_{a}=0$, the nominal case is set. Therefore $\Delta_{a}$ is a deviation on the 


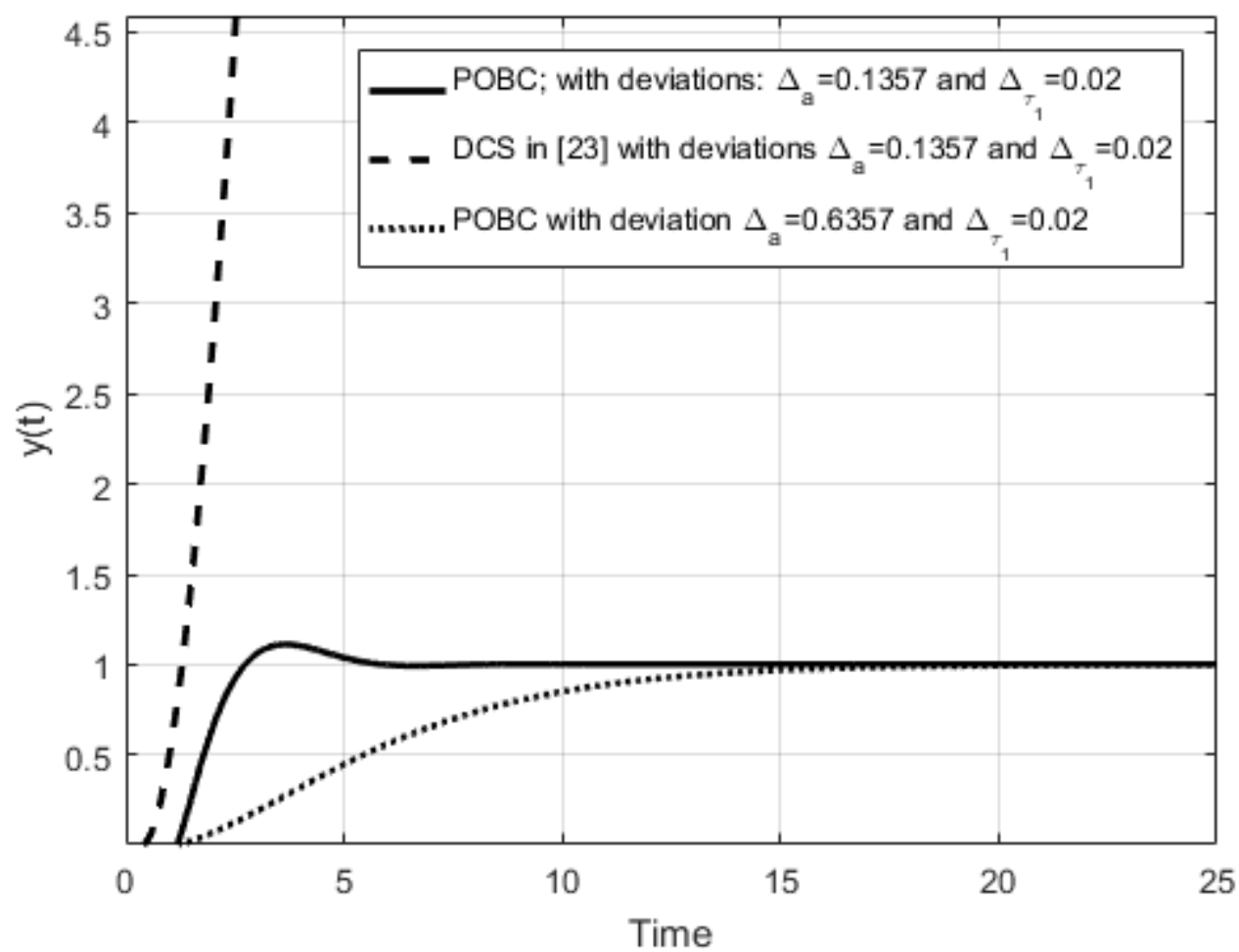

Figure 15: Output performance for uncertainties at direct loop $G_{d}(s)$.

nominal parameter, same criteria is used for the other two defined uncertainties. In Figure 15, output is shown under uncertainties into direct loop $G_{d}(s)$. This is, deviations $\Delta_{a}=0.1357$ and $\Delta_{\tau_{1}}=0.02$ are considered, which corresponds to $+20 \%$ and $+13 \%$ of uncertainty, respectively. As Figure 15 shows, under these conditions, DCS is unstable while POBC remains stable. Moreover, Figure 15 shows the output performance when POBC is submitted to uncertainties of $+95 \%\left(\Delta_{a}=0.6357\right)$ and $+13 \% \quad\left(\Delta_{\tau_{1}}=0.02\right)$ with the corresponding parameter. Therefore, POBC is robustly stable with the deviations $\Delta_{a} \in[0,0.6357]$ and $\Delta_{\tau_{1}} \in[0,0.02]$. Figure 16 shows the output performance for uncertainties into the recycling path $G_{r}(s)$. Thus, a compraison of the output is considered with uncertainties of $+222 \%\left(\Delta_{\tau_{2}}=0.22\right)$ and $+333 \%\left(\Delta_{\tau_{2}}=0.42\right)$ in the recycling delay-time. From Figure 16, POBC is robustly stable with $\Delta_{\tau_{2}} \in[0,0.42]$, while for the case $\Delta_{\tau_{2}}=0.42$ DCS is unstable.

\section{Conclusions}

The analysis developed through this work gives a stabilization strategy for a particular kind of recycling system. The proposed stabilization scheme allows the use two different controllers, i.e., a Proportional-Derivative controller and a Proportional-Integral-Derivative controller with a proposed observer strategy. Explicit stability conditions of the proposed controller as well as the observer design are obtained in terms of the system model parameters. Even when a classical PID controller has the characteristic of step tracking reference and step disturbance rejection, in this work the used PID controller does not satisfies the mentioned properties and in this case only assures closed-loop 


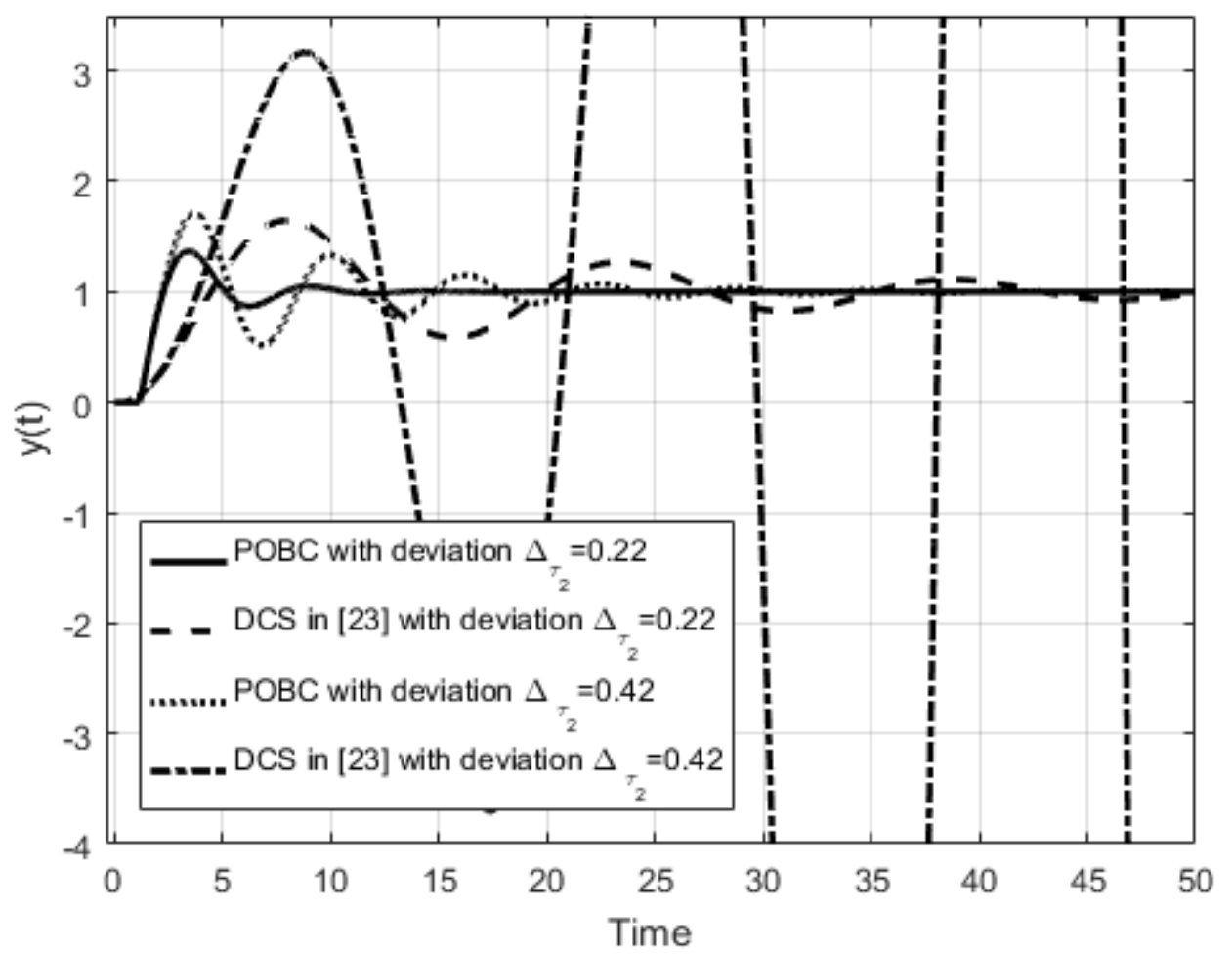

Figure 16: Output performance for uncertainties at direct loop $G_{r}(s)$.

stability, perhaps this is the consequence of dealing with a complex class of recycling systems, where the two paths consider unstable dynamics. The importance of this work arises from the fact that the proposed strategy simplify the stability problem with two different time-delay terms to a problem with only one delay term. It should be highlighted that the proposed control scheme can be applied to the case of the stable recycling system i.e., $G_{1}(s)$ and/or $G_{2}$ (s) being stable subsystems. As a future work it should be interesting to extend the proposed control strategy to a class of systems without limit in the number of unstable poles in the transfer functions of the recycling system, $\mathrm{G}_{1}(\mathrm{~s})$ and $\mathrm{G}_{2}(\mathrm{~s})$. Also, the analysis of the time-delays varying case can be performed, taking into account the proposed control scheme.

\section{References}

[1] Luyben W.L., Tyreus B. D. and M. L. Luyben, Plantwide process control, McGraw Hill, New York (1999).

[2] Luyben W. L., Temperature control of autorefrigetator reactor, J. Process Control, vol. 9, (1999) pp 301.

[3] Taiwo O., The design of robust control systems for plants with recycle, Int. J. Control, vol. 43(2) (1986), pp 671.

[4] Scali C. and F. Ferrari, Performance of control systems based on recycle compensators in integrated plants, J. Process Control, vol. 9, (1999), pp 425. 
[5] Lakshminarayanan S. and H. Takada, Empirical modelling of processes with recycle: some insights via case studies, Chem. Eng. Sci., vol. 56, (2001), pp 3327.

[6] Kwok K.E., Chong-Ping. M. and G. A. Dumont, Seasonal model based control of processes with recycle dynamics, Ind. Eng. Chem. Res., vol. 40, (2001), pp 1633.

[7] Del Muro-Cuéllar B., Velasco-Villa M., Puebla H. and J. Alvarez-Ramirez, Model approximation for Dead-Time Recycling Systems, Ind. Eng. Chem. Res., vol. 44, (2005), pp 4336-4343.

[8] Samyudia Y.K., Kadiman K., Lee O.L. and I.T. Cameron, Gap metric based control of processes with recycle systems, Proccedings of ADChEM, Pisa-Italy, (2000), pp 497-502.

[9] Astrom K.J., Hang C.C. and B.C. Lim, A new Smith predictor for controlling a process with an integrator and long dead time, IEEE Trans. Aut. Control, vol. 39(2), (1994), pp 343-345.

[10] Maza-Casas L., Velasco-Villa M. and J.A. Alvarez-Gallegos, On the state prediction of linear systems with time-delays in the input and the state, Proc. 38th. IEEE Conf. On Decision and Control, USA, December, (1999), pp. 239-244.

[11] Sanz, R., García, P., and Albertos, P. A generalized smith predictor for unstable time-delay SISO systems. ISA transactions, 72, (2018) pp.197-204

[12] Marquez-Rubio J.F., del Muro-Cuéllar B., Velasco-Villa M. and D. Cortés-Rodríguez, Observer scheme for linear recycling systems with time delays, American Control Conference, San Francisco, USA, (2011).

[13] Marquez-Rubio J.F., del Muro-Cuéllar B., Velasco-Villa M., Cortés-Rodríguez D. and O. Sename, Control of delayed recycling systems with unstable first order forward loop, J. of Process Control, vol. 22, (2012), pp 729-737.

[14] Marquez-Rubio J.F., del Muro-Cuéllar B. and O. Sename, Control of delayed recycling systems with an unstable pole at forward loop, American Control Conference, Montréal, Canada, (2012).

[15] Silva G.J., Datta A. and Bhattacharyya S.P. PID controllers for time-delay systems, Birkhuser, Boston (2005).

[16] Lee S.C., Wang Q.-G. and Xiang C., Stabilization of all-pole unstable delay processes by simple controllers, Journal of Process Control, Vol. 20, (2010), pp 235-239.

[17] Hernández Pérez, M.A., Del Muro-Cuéllar, B. and Velasco-Villa, M., PID for the stabilization of high-order unstable delayed systems with possible complex conjugate poles, Asia-Pacific Journal of Chemical Engineering, Vol. 10(5), (2015) pp 687-699.

[18] Novella-Rodríguez, D.F., Del Muro-Cuéllar, B., Marquez-Rubio, J.F., Hernández Pérez, M.A., and Velasco-Villa, M., PD/PID controller for delayed systems with two unstable poles: a frequency domain approach, International Journal of Control, https://doi.org/10.1080/00207179.2017.1386326,(2018)

[19] Bamimore, A., and Taiwo, F. Recycle compensator facilitates rapid parameterization of proportional integral/derivative (PI/D) controllers for open-loop unstable recycle processes, Industrial \& Engineering Chemistry Research, 54(18), (2015), pp 5115-5127. 
[20] Pedraza-Beltrán Y., González-Najera O. and B. Del Muro-Cuéllar, Stabilization of High Order Systems with Delay Using a Predictor Schema, 52nd. Midwest MWSCAS IEEE, Cancun Mex. August (2009).

[21] C. Xiang and Q.G. Wang and X. Lu and L.A. Nguyen and T.H. Lee., Stabilization of second-order unstable delay processes by simple controllers, Journal of Process Control,Vol. 17,(8), (2007), pp 675682.

[22] Kharitonov, V.L. and Niculescu, S.-I. and Moreno, J. and Michiels, W., Static output feedback stabilization: necessary conditions for multiple delay controllers, Automatic Control, IEEE Transactions on, Vol. 50(1), (2005) pp 82-86.

[23] Vazquez-Guerra, R.J., Marquez-Rubio, J.F., Hernández Pérez, M.A., Del Muro-Cuéllar, B., Sanchez-García, J.C., Dynamic delayed controller with recycling systems with internal delays and complex poles, Asia-Pacific Journal of Chemical Engineering, Vol. 13(1), (2018). 